\title{
Erziehungswissenschaft in Deutschland - nationale Form, kulturelle Differenzen
}

\author{
Heinz-Elmar Tenorth
}

\begin{abstract}
Humboldt-Universität zu Berlin
Disziplingeschichte wird bisher vorrangig auf nationale Kontexte bezogen und wenn kulturell, dann in Sprachkulturen und Theorietraditionen diskutiert und für das Hochschulsystem erforscht, wie sie für den deutschsprachigen Bereich typisch sind. Die Geschichte der Erziehungswissenschaft in Deutschland zeigt demgegenüber, dass jenseits nationaler Einheitsformen, wie sie durch die Sprache, die Struktur der Universitäten und durch die Ordnung der Lehrerbildung erzwungen werden, weitere kulturelle und soziale Differenzen bedeutsam bleiben. Der Vortrag soll vor allem die fortdauernde Bedeutung der Konfession und die politische und gewerkschaftliche Organisation von Lehrern und Parteien als Differenzmerkmal herausarbeiten und die Mechanismen klären, wie diese Faktoren wirksam werden.
\end{abstract}

\section{VORBEMERKUNG - THEMA UND THESE}

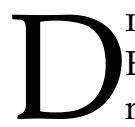
IE Disziplingeschichte der Erziehungswissenschaft argumentiert und analysiert bisher vorwiegend in nationalen Kontexten und Referenzen, auch unsere Konferenz thematisiert regionale und nationale Phänomene. Dafür gibt es gute Gründe, denn zentrale Bedingungen der Institutionalisierung der Disziplin, die ja meist den Ausgangspunkt ihrer Geschichte markiert, sind ebenso national bestimmt, nämlich durch das Wissenschaftssystem, wie offenbar bis heute auch ihre soziale Gestalt und ihre Theorietraditionen.
Selbst für die Zeit nach 1945 und in den totalitär oder demokratisch regierten Staaten Mittel- und Osteuropas dürften neben den Gemeinsamkeiten vor allem die nationalen Differenzen von Bedeutung sein. Auch grundsätzlich sind wesentliche Faktoren der Dynamik der Wissensproduktion und ihrer Referenzen innerhalb der Erziehungswissenschaft kaum zu denken, ohne z.B. nationale Bildungssysteme und die Ausbildung der Lehrerberufe in den Blick zu nehmen. Die Konzentration auf nationale Referenzen wurde auch nicht nur historisch, sondern durch die empirische Wissenschaftsforschung auch aktuell bestätigt, konnte sie doch zeigen, dass 
sich selbst Argumentationskulturen in nationaler Referenz (jetzt allerdings nach sprachlich bestimmten Ländergruppen) unterscheiden und stabil behaupten, so dass sich - mancher Gemeinsamkeiten ungeachtet, wie man sie z.B. vom Herbartianismus aus zeigen kann - eindeutige Unterschiede z.B. zwischen dem deutschsprachigen, anglophonen oder frankophonen Erziehungswissenschaften ebenso herausarbeiten lassen wie zwischen den Ländern, für die sie dauerhaft oder in bestimmten Phasen der Geschichte jeweils Vorbildfunktion hatten. Japan ist ein Beispiel dafür, wie eine nationale Erziehungswissenschaft im ausgehenden 19. Jahrhundert u.a. aus dem Kontakt mit der deutschsprachigen Erziehungsphilosophie entsteht und sich universitär entfaltet, aber nach 1970/80 zunehmend den Anschluss an us-amerikanische und anglophone Traditionen sucht.

Mit dem hier folgenden Beitrag will ich diese nationale (und sprachräumlich-regionale) Referenz für die Konstitution und Dynamik der Erziehungswissenschaft nicht etwa dementieren - und aus der Erziehungswissenschaft in Deutschland eine internationale Kommunikationsgemeinschaft ohne nationale Spezifik machen -, sondern die nationale Re- ferenz selbst präzisieren, und zwar dadurch, dass ich sie dekomponiere. Meine These ist, dass die deutsche Erziehungswissenschaft (ich ignoriere jetzt einmal die Schweiz und Österreich) in ihrem theoretischen Status und in ihrer methodisch organisierten Dynamik, z.T. auch nach ihrer Institutionalisierung, nicht angemessen verstanden ist, wenn man nicht die kulturelle Dimension mit berücksichtigt, und das heißt dann für Deutschland, dass man dann vor allem die konfessionelle und die gewerkschaftlichparteipolitische Dimension in ihrer Bedeutung sehen muss. Diese Faktoren sind für die Rolle der Religion z.T. ja auch schon analysiert worden ${ }^{1}$ (Osterwalder, Oelkers und Tenorth, 2003; Osterwalder, 1999; Tenorth, 1999; Bühler, Bühler und Osterwalder, 2013) auch für die Frühgeschichte des Denkens über Erziehung, sie bleiben aber bedeutsam auch im 19. Jahrhundert und sie sind folgenreich wirksam bis weit ins 20. Jahrhundert, sogar latent auch aktuell noch von Bedeutung, und zwar sozial wie epistemisch.

An dieser Stelle kann ich den Zusammenhang von Konfessionalität, Politik und Professionspolitik mit den Theoriestrukturen der Erziehungswissenschaft natürlich nicht insgesamt diskutieren. Ich konzentriere mich

\footnotetext{
${ }^{1}$ Ich erinnere nur an die folgenden Arbeiten: Fritz Osterwalder, Jürgen Oelkers und Heinz-Elmar Tenorth (2003, Hrsg.): Das verdrängte Erbe. Pädagogik im Kontext von Religion und Theologie. Weinheim, Basel (Beiträge zur Theorie und Geschichte der Erziehungswissenschaft Bd. 25), auch an die Diskussion von Osterwalders generalisierenden Thesen in Neue Pestalozzi-Blätter 5 (1999)2 sowie meine dortige Stellungnahme: Heinz-Elmar Tenorth: Rettungslos der Religion verfallen? Fritz Osterwalder als Propagandist säkularer Pädagogik. In: Neue Pestalozzi-Blätter 5 (1999)2, S. 21-23. Für den argumentativen Kontext relevant auch Patrick Bühler, Thomas Bühler und Fritz Osterwalder (2013,Hrsg.): Zur Inszenierungsgeschichte pädagogischer Erlöserfiguren. Haupt Verlag, Bern, und dazu meine Rezension In: http:/ / hsozkult.geschichte.hu-berlin.de/rezensionen / 2014-1-167, die zeigt, dass pädagogische Klassizität in Formen religiöser Erlöserfiguren konstruiert wird.
} 
nach eher knappen und nur einleitenden und retrospektiven Hinweisen (1.) vielmehr auf das 20. Jahrhundert, dann zuerst auf die katholische Seite (2.), danach auf die protestantische (3) und schließlich auf das latente, aber wirkungsvolle Fortleben von Religion und religiösen Denkformen im politischen und bildungstheoretischen Kontext (4.). Die Indikatoren für die hier zu diskutierende Entwicklung suche ich zum einen in institutionellen Mustern der Etablierung von Pädagogik im nationalen Bildungssystem, dann auch kommunikativ, vor allem aber in der publizistischen und sozialen Infrastruktur für die Produktion von Erziehungswissen und erziehungswissenschaftlichem Wissen und schließlich in den Produkten selbst, die dabei erzeugt worden sind, d.h. in deren theoretischen Eigenarten, Prämissen und Argumenten.

\section{Bildungssystem, Profession Und Konfession - DIE TRAdITIONSLINIEN BIS 1933}

Einige knappe historische Erinnerungen sollen die große Bedeutung der Konfessionsreferenz erläutern, und zwar für die soziale und theoretische Konstitution der Pädagogik als Wissenschaft und auch für die damit unmittelbar verbundene Organisationsfrage der Lehrerberufe und ihre professionelle Identität. Diesterweg (1852) z.B., um im 19. Jahrhundert einzusetzen, beschreibt den Weg der Emanzipation des Lehrers und der Pädagogik als Weg von der „Kirchenlehre zur Pädagogik" und entspre- chend definiert er - folgenreich bis in den liberalen Deutschen Lehrerverband (DLV) bis 1933, und die Gewerkschaft Erziehung und Wissenschaft (GEW) bis heute - die Organisationsfrage für den Lehrerberuf als Prozess der Emanzipation von fremden Gewalten. Er ignoriert und toleriert dabei aber die Konfessionalität der Lehrerbildung und handelt sich zugleich den christlich-nationalen Etatismus ein, der für den liberalen Protestantismus in Deutschland im Bündnis von Thron und Altar, Bildungssystem und Bildungstheorie typisch war und dort als theoretisch legitim begründetes Verhältnis zur Welt ausgegeben wurde. Die Fixierung auf den Staat wurde zumindest im streng protestantischen Milieu schon historische als Fehler codiert und mit einem staats- bzw. bürokratiekritischen Modell öffentlichen Unterrichts konfrontiert, in dem das Bildungssystem und die Identität der Profession anders definiert wurden Dörpfeld mag dafür als Beleg heute aus reichen. Aber die Alternative bekräftigte den Konfessionalismus eher als dass sie ihn abschwächte, denn die Alternative wird zugunsten der alten Kirche und ihrer autonomen Gemeinden entwickelt, religiös traditional und konservativ, wie manche Rezipienten in ihrer Freude an dieser Staats - und Bürokratiekritik gern vergessen. Zum Glück haben die staatskritischen liberalen und laizistischen demokratischen Opponenten des 19. Jahrhunderts, allen voran der neukantianische Philosoph und Schulmann 
Friedrich Albert Lange ${ }^{2}$, diese schlechte Pointe gesehen und scharf kritisiert (vgl. Tenorth, 1989).

Auf der katholischen Seite ist der Primat des Konfessionellen nicht so konflikthaft gespalten, sondern bis ins 20. Jahrhundert eindeutig, bevor auch hier - nach 1960 - innerkirchliche Kritiker des Konfessionsprinzips auftreten. Zunächst hat - theoretisch und professionspolitisch - z.B. der rheinische Schulmann und Lehrerbildner Lorenz Kellner (1811-1892) ${ }^{3}$ im 19. Jahrhundert eine vergleichbare Bedeutung wie Diesterweg. Auch die katholischen Verbände für Lehrerund Lehrerinnen und die von gepflegte theoretische Pädagogik sind vergleichbar gut identifizierbar (und dann theoretisch eher nah bei Pestalozzi und Diesterweg als bei Dörpfeld - ohne den preußischen Staat, zumal den des Kulturkampfes - umstandslos zu akzeptieren) (Cloer, 1977; Heinemann, 1977). Die eigene Systematik einer katholischen Pädagogik, fehlt aber nicht. Sie schreibt im ausgehenden 19. Jhdt. Otto Willmann, in seinem Alterswerk, aristotelischthomistisch und als Weisheitslehre,

${ }^{2}$ Vgl. Heinz-Elmar Tenorth (1989): Die Last der Autonomie. Über Widersprüche zwischen Selbstbeschreibungen und Analysen des Bildungssystems seit dem 19. Jahrhundert. In: K.-E. Jeismann (Hrsg.): Bildung, Staat, Gesellschaft im 19. Jahrhundert. Wiesbaden 1989, S. 413 - 431 sowie die Daten zu Langes Biographie: Lange seit 1841 in Zürich, Studium 1848-1851 in Bonn (Theologie, Philosophie und Philologie), Dr. phil. 1851 (Questiones Metricae), von 1852 bis 1855 Gymnasiallehrer in Köln, ab 1857 Privatdozent für Philosophie und Pädagogik in Bonn, bis 1861 Gymnasiallehrer Duisburg. Aus Protest gegen die Politik Bismarcks trat Lange 1862 aus dem Schuldienst aus, weil er sich gegen ein königliches Rundschreiben wehrte, das Lehrer von jeglicher politischer Agitation abhalten sollte. Nach 1862 publizistische Tätigkeit in Duisburg, seit 1866 Gymnasiallehrer in Zürich bzw. Winterthur, 1869 Habil. Philosophie an der Universität Zürich, dort auch 1872-1875 Prof. für Philosophie, 1875 in Marburg a.d. Lahn gestorben.

${ }^{3}$ Lorenz Kellner: 1828-1831: (protestantisches!) Lehrerseminar Magdeburg (bei Zerenner!), 18311836 Lehrer an Volksschulen in Mackenrode und Erfurt, dort ab 1833 Rektor; 1836-1848 Heiligenstadt (an seines Vaters Arbeitsort), 1848 Regierungs- und Schulrat im Kreis Marienwerder (Westpr.), 18481849 Mitglied des Preußischen Abgeordnetenhauses (wie Diesterweg); 1855-1886 Regierungs- und Schulrat in Trier, 1867-1870 Abgeordneter des Wahlkreises St. Wendel im Preußischen Landtag. 1863 Dr.phil.h.c. Akademie Münster, 1871 Geh. Regierungsrat, seit 1872 Mitglied der preuß. Kommission für die Beratung des Volksschul- und Seminarwesens (Plan Falk). Im Kulturkampf trotz seiner kirchentreuen Einstellung infolge guter Beziehungen zu den Trierer Regierungspräsidenten unangetastet. Der Katholische Lehrerverband ernannte ihn 1891 zum Ehrenmitglied. (vgl. zu diesen Daten E.M.Roloff: Lorenz Kellner. In: Lexikon der Pädagogik, 2. Bd., Freiburg 1913, Sp. 1163-1170, und dort zur Qualifizierung seiner Bedeutung: "der hervorragendste der praktischen katholischen Pädagogen der Neuzeit“ (1167). Schriften: "Aphorismen zur Pädagogik“ - 1850, 17. Aufl. 1907; "Volksschulkunde" - 1855, ${ }^{8} 1886$, „Was K.s Schriften ... besonders auszeichnet, ist die ausgereifte, wohltuende Ruhe $\mathrm{u}$. Überlegenheit, die sie dem Leser als eine Labsal empfinden lässt zu einer Zeit, in der das aufdringliche $\mathrm{u}$. verworrene Phrasentum moderner Überpädagogen die Welt mit seinen Disharmonien u. Bizarrerien erfüllt." (1170)

Weitgehend vergessen, wird Kellner jüngst neu entdeckt bei Patrick Bühler: Stets „conservativ katholisch ${ }^{\prime}$ Lorenz Kellners moderne Pädagogik. In: Bühler u.a.: Zur Inszenierungsgeschichte pädagogischer Erlöserfiguren. 2013, S. 225-240 - mit der These, dass der Autor und seine politische Position katholisch gewesen sein mag, seine Pädagogik, in der Pestalozzi-Nachfolge, als „pädagogische Opfer- und Liebessemantik" (235) dem mainstream der Zeit aber entsprochen habe, weder reformiert noch katholisch, sondern wie alle eine „diffus christliche Innerlichkeitspädagogik ..., die ... als genuin katholisch reklamiert wird" (ebd.) 
nachdem er sich von der "Socialforschung" verabschiedet hatte ${ }^{4}$ (Brezin$k a$, 2003). Danach gibt es den einschlägigen Versuch von Joseph Göttler (1915) ${ }^{5}$, Professor an der Katholischen Philosophisch-Theologischen Hochschule in Freising, dann in der Theologischen Fakultät der Universität München, dessen „System der Pädagogik" Neuauflagen noch nach 1950 erlebt (die 12. Auflage stammt von 1964) (Göttler, 1915). ${ }^{6}$ Auch dieses Disziplinkonzept mischt Bekanntes und Eigenes: Pädagogik ist bei ihm natürlich, wie sonst meist auch, eine "normative“ Wissenschaft (14f.), die er dann methodisch zu den Geisteswissenschaften rechnet (15f.), die ja auch eine normative Basis hatten. Die katholische Lehre reicht aber bis in die Grundbegriffe hinein: die grundbegrifflich für die Pädagogik seit Herbart zentrale Bildsamkeits-Thematik wird u.a. von "Erbsünde und Gnade" aus erläutert (S.98f.), "Gott" gilt in einem ganz umfassenden Sinne "als Urquell aller Erziehungsautorität und als Erziehungsfaktor“, denn „in seiner Hand liegt die Wirksamkeit aller Erzieher und Erziehungsfaktoren ... er wirkt nicht neben, sondern in ihnen allen und durch sie." (S. 133ff.) Staat und Kirche werden als „Erziehungsgewalt und Erziehungsfaktor" (S. 135ff.) einander konfrontiert, die beide - wenn der Staat nur „Kulturstaat" (136) ist - ein Erziehungsrecht haben, mit dem Primat der Kirche in Sachen der Religion und Sittlichkeit, neben Familie, Natur und Kultur. Nicht nur institutionell, auch theoretisch gibt es also eine katholische Pädagogik, philosophisch und systematisch vor allem, und natürlich auch eine kirchennahe protestantische, von Palmer bis Schmid im 19. Jahrhundert, später auch in den Thesen von Kittel bis Delekat auch im 20. Jahrhundert. Diese Pädagogik gesteht dem Staat ein eigenes Erziehungsrecht erst nach $1950 \mathrm{zu}$ und kämpft genauso wie die Katholische Pädagogik für das Konfessionsprinzip in der Elementarschule und ihrer Lehrerbildung (vgl.

\footnotetext{
${ }^{4} \mathrm{Zu}$ Willmann jetzt Wolfgang Brezinka: Pädagogik in Österreich. Die Geschichte des Faches an den Universitäten vom 18. bis zum Ende des 20. Jahrhunderts. Bd. 2: Pädagogik an den Universitäten Prag, Graz und Innsbruck. Wien 2003, S. 21ff., auch für den Wandel „zu einem katholischen Weltanschauungsphilosophen" (38), der eine „Weisheitslehre" aufbaut, die Wissensbestände rezipiert, ohne Theoriestandards zu genügen: „ohne zwischen rationalen Erkenntnissen und Glaubensüberzeugungen, zwischen Wissenschaft, Philosophie und Weltanschauung klar zu unterscheiden. ... dominiert breites historisches Normen- oder „Prinzipienwissen" aus natürlichen und übernatürlichen Quellen der „Weisheit" in Gegnerschaft zu Aufklärung, Modernismus, Neuheidentum und „positivistischer" Wissenschaft" (so Wolfgang Brezinka, unpubliziertes Ms. 2015, S. 18).

${ }^{5}$ Joseph Göttler: 1874-1935, 1898 kath. Priester, 1904 Habil. und 1909 Prof. Theologische Hochschule Freising, 1911 Prof. Pädagogik und Katechetik, Univ. München. Klaus-Peter Horn: Erziehungswissenschaft in Deutschland im 20. Jahrhundert. Bad Heilbrunn 2003 nennt Göttler nicht, sondern rechnet ihn der Theologie zu (vgl. S. 28 sowie 67, Anm. 147), für die Wirkung in der Disziplin nennt er allerdings Göttlers Assistenten bis 1935, Joseph Dolch (Horn 2003, S. 28, 131, 215).

6 Joseph Göttler: System der Pädagogik. (1915), neu bearb. und erw. von Johann B. Westermayr [Prof. an der Philosophisch-Theologischen Hochschule Freising]. München ${ }^{7} 1947$, im Verlag Kösel erschienen, der neben dem Herder-Verlag in Freiburg und dem Verlag Auer in Donauwörth zu den für die katholische Pädagogik wichtigen Verlagen gehört. Pädagogik gilt für Göttler natürlich als eine „normative“ Wissenschaft (14f.), die er methodisch zu den Geisteswissenschaften rechnet (15f.).
} 
Müller-Rolli, 1999). ${ }^{7}$

Politisch, für die andere Seite meiner Dekomposition, wird die Theoriefrage von Erziehung und Erziehungsreflexion spätestens seit der Revolution von 1848 geschrieben, wo sie natürlich als "Staatspädagogik" und etatistisch bereits vorgefunden wird, dann aber in kritischer Wendung und in der Absicht der Autonomisierung gegen den Staat, gelegentlich auch laizistisch, immer aber gegen die staatsgestützte Kirche und ihre Rolle im Bildungswesen. Solche Argumente finden sich früh nicht nur bei Marx, auch bei Weitling in der Revolution von 1848 und vorher schon in den bekannten diversen Utopien. Professionspolitisch folgenreiche Positionen der Sozialisten dominieren dabei. Liberale und demokratische Theoretiker wie etwa Friedrich Albert Lange haben in der Theorie oder in der Lehrerschaft und auch im Staat keine Anhänger, auch die radikalen Anarchisten nicht. Die Positionen der Sozialisten können sich an den Universitäten bis 1918 natürlich nicht ausbreiten, sie werden organisatorisch auch innerhalb der Lehrerschaft erst seit dem frühen 20. Jahrhundert vertreten (Breyvogel, 1977; Trube, 1977, Hoffmann 1977; Stöhr, 1978) ; dann aber breit in allen Lehrergruppen bis zu den Gymnasialund Hochschullehrern; der „Bund Entschiedener Schulreformer ${ }^{\prime 8}$ mag als Beleg reichen (Uhlig, 1979). Die hier, im sozialistischen Milieu, propagierten bildungspolitischen Positionen - in der Ordnung von Weltlichkeit und Staatlichkeit, Unentgeltlichkeit und Einheitlichkeit - entstammen zwar der parteipolitischen sozialistischen Kritik der Schule des Untertanen und der dazugehörigen kritischen Argumentation der Leh$\operatorname{rer}^{9}$ (Sack, 1961; Günther, 1988; Rang, 1982) sie werden aber schon bald einer theoretischen Veredelung und argumentativen Verselbständigung zugeführt. Frühe Versuche einer Soziologie der Erziehung verdanken sich ebenso

\footnotetext{
${ }^{7}$ Vgl. die einschlägigen Dokumente und Kommentare in: „Evangelische Schulpolitik in Deutschland 1918-1958. Dokumente und Darstellung. Hrsg von Sebastian Müller-Rolli unter Mitarbeit von Reiner Anselm und einem Nachwort von Karl Ernst Nipkow. Göttingen 1999.

${ }^{8}$ Dazu gehört ja nicht nur der Gymnasiallehrer Paul Oestreich, sondern auch bekannte Hochschullehrer, vgl. die Literatur seit Winfried Böhm: Kulturpolitik und Pädagogik Paul Oestreichs. Bad Heilbrunn 1973 bis zu Christa Uhlig: Theoretische Auffassungen Paul Oestreichs zur Entschiedenen Schulreform . In: Jahrbuch für Erziehungs- und Schulgeschichte 19(1979) S.53-67; die Arbeiten von Reintges oder Eierdanz sind weniger empfehlenswert.

${ }^{9}$ Exemplarisch dafür Eduard Sack: Die preußische Schule im Dienste gegen die Freiheit. Schulpolitische Kampfschriften. Ausgew. und eingel. von K.-H.Günther. Berlin 1961; für die Rezeption der alten Utopien, der ,fortschrittlichen' bürgerlichen Theorie und der neuen Sozialisten von Liebknecht zu Neubauer oder Oestreich, in der DDR-Tradition u.a. Karl-Heinz Günther: Über pädagogische Traditionen. Berlin 1988. Solche Analysen werden zugleich als Texte zur Theorie der Erziehung und als soziologische Analyse ausgegeben und lösen entsprechende Theoriekontroversen in der DDR aus, wie Brita Rang gezeigt hat: B.R.: Pädagogische Geschichtsschreibung in der DDR. Frankfurt a.M./New York 1982. Rang zeigt, dass in der DDR nach 1945/50 in der pikanten Konstellation diskutiert wird, dass man die erziehungssoziologischen Positionen des Nationalsozialisten Ernst Krieck und des Kommunisten und Nazi-Opfers Robert Alt über die gesellschaftliche Funktion von Erziehung gegeneinander abgleichen muss, wenn man kritische Geschichte der Erziehung schreiben will.
} 
diesem angelagerten Theoriekontext wie Studien zur Berufsbildung oder zur Lage der Kinder oder zum Klassencharakter des Bildungswesens und zur Realisierung oder Behinderung von Bildungschancen. Man findet diese Thesen - deutschsprachig - in den Schriften von Rühle bis Kanitz, von Löwenstein bis Glöckel, von Anna Siemsen bis Hoernle, von Alfred zu Max Adler et.al. ${ }^{10}$, und natürlich ist auch Bernfeld $^{11}$ (1925) nicht zu vergessen, auch wenn seine Reflexionen deutlich theoretischer ambitioniert sind. Die Argumente bleiben bis heute, wenn auch deutlich gemildert, in der Bildungspolitik der SPD präsent, parteihistorisch gut dokumentiert in den Texten von Heinrich Schulz bis Clara Zetkin und Konrad Haenisch (1921). Sie sind auch virulent in den Abgrenzungsund Ausschließungsprozessen, die bis zum Ausschluss der Jungsozialisten und zum Godesberger Programm dieser Theorie-, Reflexions- und Agitationsarbeit parallel gehen. Diese Ideen werden zugleich aber sowohl schulpolitisch als auch erziehungstheoretisch von Leonard Nelson weiter angereichert und von Heinz-Joachim Heydorn philosophisch veredelt (gelegentlich vielleicht auch nur in Schwundstufen tradiert). Sie bleiben dadurch bedeutsam bis in die Debatten über Schulreform, Schulstruktur und Chancengleichheit in der Bildungsreformeuphorie noch nach 1970 und in der GEW und bei gesellschaftskritischen Kollegen - man lese die Texte von Gruschka bis Sünker - hier und da bis heute. Um 1970 galten sie als Theorie, heute eher als politische Statements, aber mit ihren Thesen bestimmen sie immer noch die Frage mit, wie innerhalb der Pädagogik über "Gleichheit" diskutiert wird. Denn das geschieht nach wie vor relativ untheoretisch, z.B. schon, ohne pädagogische und gesellschaftliche Gleichheit zu unterscheiden. Aber die alten Thesen beflügeln das politisch-praktische und kritische Selbstverständnis großer Frak-

\footnotetext{
${ }^{10}$ Die Studentenbewegung und die Pädagogik haben diese Texte nach 1970 neu entdeckt und ediert, vgl. u.a. (nur als Auswahl): Alice und Otto Rühle: Erziehung und Gesellschaft. Berlin 1972 (Raubnachdruck der fünf Hefte der Zeitschrift „Am andern Ufer - Blätter für sozialistische Erziehung. Dresden/Leipzig (1924-1926) - mit dem ehrgeizigen Versuch "Gesellschaftsleben und Seelenkunde, Marxismus und Individualpsychologie (zu) verbinden“ (zit. S. 15); Otto Rühle: Zur Psychologie des proletarischen Kindes. (1925) Hrsg. Von L.v.Werder und R.Wolff, Frankfurt a.M. 1969; Edwin Hoernle: Grundfragen proletarischer Erziehung. (1929 - und andere Texte) Ffm. 1969/tbAusgabe 1973; Otto F. Kanitz: Das proletarische Kind in der bürgerlichen Gesellschaft. (1925 u.a. Aufsätze) Ffm. 1970; Kurt Löwenstein: Sozialismus und Erziehung. Eine Auswahl aus den Schriften 1919-1933. Hrsg. Von F.Brandecker/H.Feidel-Merz. Berlin/Bonn 1976; Anna Siemsen: Beruf und Erziehung. Berlin 1926; dies: Die gesellschaftlichen Grundlagen der Erziehung. Hamburg 1948 sowie, für manche weniger beachtete Autoren, die Analysen und Quelleneditionen für die Zeit bis 1918 in Christa Uhlig: Reformpädagogik: Rezeption und Kritik in der Arbeiterbewegung. Frankfurt a.M. 2006.

11 Exemplarisch steht für seine theoretisch andauernde Geltung natürlich Siegfried Bernfeld.: Sisyphos oder die Grenzend er Erziehung. Wien 1925. Für die Studentenbewegung war die dreibändige Edition von Lutz von Werder und Reinhart Wolff inspirierend (Antiautoritäre Erziehung und Psychoanalyse. 3 Bde, Frankfurt a.M. 1969), Ulrich Herrmann ediert z.Zt. das Gesamtwerk von Siegfried Bernfeld (vgl. „Sämtliche Werke“, zuerst Weinheim/Basel, jetzt Gießen, seit 1991, z.Zt. 8 Bde, geplant 11 Bde).
} 
tionen einer Erziehungswissenschaft, die sich selbst als „kritisch“ versteht.

Nicht nur bei den Lehrerverbänden nach 1900 haben diese Texte auch für lange Zeit die Annahme bekräftigt, dass zwischen einer Bildungspolitik ihren Musters und der (empirischen) Erziehungswissenschaft ein natürliches, systematisch begründetes KonsensVerhältnis besteht. Ernst Meumann hat mit seinen Vorträgen diesem Verständnis ebenso Vorschub geleistet wie andere Teile der Reformpädagogik oder die „Erziehungswissenschaftliche Hauptstelle des Deutschen Lehrervereins" (Tenorth, 2013). Dieses Bündnis von Erziehungswissenschaft und Lehrerbewegung besaß durchaus auch Macht im Wissenschaftssystem: Der Lehrstuhl von Ernst Meumann in Hamburg z.B. kann nach dessen Tod 1916 nur mit Beteiligung und Zustimmung der örtlichen Lehrer (in der „Gesellschaft der Freunde des vaterländischen Schul- und Erziehungswesens" organisiert, wie die seit 1805 bestehende Vorläuferorganisation der GEW früher hieß) besetzt werden. Spranger sagt deswegen entrüstet $a b$, weil er den Streit mit den Lehrern und deren politische Macht, die theoriestrategisch im Verweis auf empirische Forschung und Reformpädagogik begründet und auch theoretisch gegen ihn gewendet wurde, schon aus Leipzig kannte und in der Kritik seiner Lehrpraxis und Bildungspolitik bereits leidvoll erfahren hatte.
Das wollte er sich nicht noch einmal antun (und die Hamburger handeln sich neben dem erst 1929 kommenden Wilhelm Flitner - 1923 zuerst Gustav Deuchler ein, der zwar empirisch forschte, sich aber auch bald den $\mathrm{Na}-$ tionalsozialisten zuwandte) (Scheuerl, 1991).

Aber Sprangers Pädagogik war natürlich selbst nicht frei von Politik, sondern staatsfixiert, preußisch und protestantisch (Himmelstein, 2013) und das führt zurück in die Konfessionsfrage, jetzt nach 1920. Die Fortsetzung der Konfessionalität der Elementar-Lehrerbildung innerhalb der Lehrebildung generell und auch in den neu gegründeten Pädagogischen Akademien nach 1918/20 schreibt zunächst in diesem Revier auch theoretisch die alten Verhältnisse der konfessionsorientierten Spaltung der begleitenden Reflexion fort. Selbst die universitären Referenzen sind eindeutig konfessionell geprägt, wenn auch nicht so manifest. Neben den in Mittel- und Norddeutschland dominierenden Protestanten haben die Katholiken z.B. in Preußen nach 1810/14 erst nur in Bonn und Breslau, später auch in Münster, ihre Ausbildungs- und Rekrutierungsorte, weil man schlecht ignorieren konnte, dass es katholische Preußen gab, die auch der Erziehung, einschließlich der Universitäten, bedurften; diese Repräsentanz in Preußens Universitäten wurde 1919 von Konrad Adenauer um Köln erweitert. Die Katholiken haben

\footnotetext{
${ }^{12}$ Dort dann auch mit den Konkordatsproblemen, vgl. dazu Marjorie Lamberti: The Politics of Education. Teachers and School Reform in Weimar Germany. New York/Oxford: Berghahn 2002 /paperback: 2004.
} 
natürlich Bayern und Baden (Lamberti, 2004) ${ }^{12}$ Diese Zuschreibung konfessioneller Reviere gilt auch nicht nur institutionell, universitär und für die Elementarlehrer, sondern auch kommunikativ und theoretisch für die gesamte Disziplin.

Man kann die Wirkungen dieser Lage in der begrifflichen und semantischen Opposition von „Erziehungswissenschaft" vs. „Geisteswissenschaftliche Pädagogik" beobachten. Die Frage der Konfessionalität reicht als bis in den Kern der Disziplin hinein und zeigt dort die Folgen des Schismas zwischen katholischer und protestantischer Pädagogik. Eine solche konfessionsbasierte grundlagentheoretische Stilisierung des Schismas ist auch trotz der Tatsache möglich, dass die protestantische Seite auf den Universitäten sich selbst nicht als protestantisch versteht, sondern als rein wissenschaftlich - das werde ich noch diskutieren. Publizistisch ist die Sachlage aber ganz eindeutig: Das katholische Sammelwerk über das disziplinäre Wissen erscheint als (unvollendetes) „Handbuch der Erziehungswissenschaft" (1928-1934) ${ }^{13}$, die preußisch-protestantische Pädagogik sammelt sich im „Handbuch der Pädagogik" ${ }^{14}$ von Herman Nohl und Ludwig Pallat (1928-1933), einem leitenden Mitarbeiter des staatlichen preuBischen Zentralinstituts für Erziehung und Unterricht; das katholische "Lexikon der Pädagogik der Gegenwart “15 wiederum, ediert von Karl-Josef Spieler (1930-32), konkurrierte mit dem „Pädagogischen Lexikon“ (1928-1931), das Hermann Schwartz ${ }^{16}$ im Auftrag des „Vereins für evangelische Pädagogik" herausgegeben hat. Bei den Zeitschriften ist die konfessionelle Fraktionierung nicht anders: Im Herausgeberkreis der „Erziehung“ sitzt niemand, der auch katholische Zeitschriften oder vergleichbare Standardwerke ediert, und die „Vierteljahrsschrift für wissenschaftliche Pädagogik" ist natürlich auch frei von Protestanten und belegt noch einmal die Randstellung katholischer Pädagogik; denn ihre Au-

\footnotetext{
${ }^{13}$ Handbuch der Erziehungswissenschaft. Hrsg. im Auftr. d. Dt. Inst. f. wiss. Pädagogik, Münster i. W., v. Franz Xaver Eggersdorfer, Max Ettlinger, Josef Schröteler [u. a.], 4 Bde, München (Kösel) 1928-1934. Von den geplanten fünf Teilen und insgesamt 28 Bänden (vgl. Müller S. 92ff.) sind 11 Bände erschienen: Thematisch präsentiert es die folgende Ordnung der Erziehungswissenschaft: 1. Teil (geplant: 4 Bde): Allgemeine Erziehungslehre. II. Teil (3 Bde), Pädagogische Hilfswissenschaften; III. Teil (7 Bde): Pädagogik und Didaktik der Altersstufen; IV. Teil (10 Bde): Besondere Bildungslehre; V. Teil (4 Bde): Geschichte der Erziehung und der Theorie der Erziehung..

${ }^{14}$ Herman Nohl/Ludwig Pallat (Hrsg.): Handbuch der Pädagogik. 5 Bde und Register, Langensalza (Beltz) 1928-1933, 1. Bd.: Die Theorie und die Entwicklung des Bildungswesens (1933); 2. Bd.: Die biologischen, psychologischen und soziologischen Grundlagen der Pädagogik (1929); 3. Bd.: Allgemeine Didaktik und Erziehungslehre (1930); 4. Bd.: Die Theorie der Schule und der Schulaufbau (1928); 5. Bd.: Sozialpädagogik (1929).

15 Karl Josef Spieler (Hrsg.): Lexikon der Pädagogik der Gegenwart. 2 Bde., Freiburg (Herder) 1930/1932 „im Lichte katholischer Weltanschauung“ (Vorwort) - und der Herausgeber war im Übrigen Mitglied der NSDAP (Müller, S. 89, Anm. 180) - zu den Autoren ebd., S. 90 und Anm. 185, wo die "Vielschreiber" genannt werden, von denen niemand auch außerkatholisch schreibt, viele zwar promoviert, aber nicht im Universitätssystem selbstständig lehrend tätig sind.

16 „Pädagogisches Lexikon“, Hrsg, von Hermann Schwartz in Verbindung mit der Gesellschaft für evangelische Pädagogik. Bielefeld, Velhagen \& Klasing, 4 Bde (alphabetisch geordnet), 1928-1931.
} 
toren sind deutlich weniger an den renommierten norddeutschen Universitäten vertreten (etc.).

Zwischen den konfessionell markierten kommunikativen Netzwerken gibt es im Alltag der Wissensproduktion so gut wie keine Überschneidungen. ${ }^{17}$ Man stößt vielmehr auf geschlossene Zirkel der Kommunikation, die sich auch milieuspezifisch und theoriebestimmt unterschiedlich rekrutieren und - trotz mancher Überschneidungsmengen - auch systematisch und argumentativ abgrenzend definieren. Denn sie codieren auch die Erziehungswissenschaft bzw. die wissenschaftliche Pädagogik anders, modern und erfahrungswissenschaftlich bei den Katholiken, dann auch in Verwendung des Begriffs Erziehungswissenschaft, wofür Joseph Dolch (1933), Assistent bei Göttler, Beleg ist ${ }^{18}$, geisteswissenschaftlich, traditional und in der Abwehr der Erziehungswissenschaft innerhalb des protestantischen Milieus -, wie man in der
Nohl-Schule oder bei Spranger sehen kann, die sich damit zugleich von den Lehrergewerkschaften und deren Wissenschafts- und Bildungspolitik, distanzieren. „Erziehungswissenschaft", so habe ich Wilhelm Flitner selbst noch in Tübingen in den 1970er Jahren klagen gehört, ,"das hat uns die Gewerkschaft eingebrockt".

Einzig Aloys Fischer (1880-1937, katholisch) lässt sich nicht eindeutig zuschreiben, sondern kann mit jeder Konfession, aber auch mit den unterschiedlichen theoretischen Schulen, empirisch oder geisteswissenschaftlich, selbst mit den Soziologen und Philosophen (die sonst nur Litt, Spranger oder Nohl akzeptieren). Fischer arbeitet auch praktisch und politisch sowohl mit dem Staat - in Bayern- wie mit den reformpädagogischen Bewegungen, deren Hauszeitschrift er zusammen mit Otto Scheibner (evangelisch) edierte ${ }^{19}$. Aber die Ausnahme bestätigt nur die Fraktionierung, die Aloys Fischer ja auch selbst als Zersplit-

\footnotetext{
17 Aber natürlich gibt es einzelne Ausnahmen: Über die Geschichte der katholischen Bildung schreibt in Bd. 1 von Nohl\&Pallat Siegfried Behn. Jonas Cohn, eine Sonderfigur im nicht-preußischen Milieu, schreibt über Aufklärung und Idealismus. In Bd. 2 kann man lernen, dass Ernst Krieck zum Nohl-Milieu gehört, in Bd. 3 schreibt der Jesuit Dunin-Borkowski über katholische religiöse Erziehung, in Bd. 4 schreibt bildungspolitisch folgenreich tätige Katholik Eugen Löffler (Stuttgart) über die Schulreform in den Ländern, die Sozialpädagogik in Bd. 5 ist fest in protestantisch-preußischer Hand.

Umfassende statistische Analysen dieser Netzwerke, basierend auf den Autoren der nachstehend genannten Handbücher und Lexika, den Herausgebern der einschlägigen Zeitschriften und der Auswertung publizistischer Kooperationen für die Zeit bis 1933 verdanke ich u.a. Swetlana Nordheimer und Thomas Baldauf - und der Förderung durch die DFG.

18 Joseph Dolch: Erziehung und Erziehungswissenschaft in Deutschland und Deutschösterreich von 1900 bis 1933. München 1933; ders.: Lesebuch zur Erziehungswissenschaft. 1940; noch nach 1945 - ders.: Aufgaben und Aufbau der Pädagogik, einer umstrittenen Wissenschaft. (1952) ND in: H.Röhrs (Hrsg.): Erziehungswissenschaft und Erziehungswirklichkeit. Frankfurt am Main 1967, S. 123-136.

${ }^{19}$ Scheibner (1877-1961) wiederum war 1910 Mitgründer die „Zeitschrift für pädagogische Psychologie, experimentelle Pädagogik und jugendkundliche Forschung" und Schriftleiter der Zeitschrift „Die Arbeitsschule“ seit 1923, deren Mitherausgeber Aloys Fischer war (zusammen mit Georg Kerschensteiner, Ludwig Pallat und Richard Seyfert).
} 
terung und damit als Theorie- und Statusproblem der wissenschaftlichen Pädagogik auf dem Kongress über „Neue Erziehung"1928 kritisch notiert hat (Fischer, 1928).

Diese Fraktionierung basiert auf auch seit langem etablierten, eindeutig zurechenbaren materialen kommunikativen Infrastrukturen: Die Handbücher und Zeitschriften haben ihre eigenen Verlage, nahe beim protestantischen Milieus wie Beltz oder Klinkhardt, Quelle \& Meyer oder Velhagen \& Klasing, und natürlich, auf der Gegenseite, auch die katholischen: Herder in Freiburg natürlich, auch bei Pustet oder Kösel in München oder bei Auer in Donauwörth. Das preußische Kultusministerium publizierte in der Weidmannschen Buchhandlung in Berlin, die Schriften des Zentralinstituts für Erziehung und Unterricht erscheinen bei Mittler \& Sohn (Tenorth, 1996) und dort werden auch seine reformpädagogischen Aktivitäten betreut, z.B. die Erörterung der Sexualpädagogik. ${ }^{20}$ Die katholische Seite dagegen publiziert natürlich, wenn auch etwas später, zum selben Thema ebenfalls, allerdings im „Pädagogischen Verlag“ in Düsseldorf (und nicht ohne Imprimatur des Kölner erzbischöflichen General- vikariats) $^{21}$ (Schröteler, 1921).

Diesen Text wie andere betreut das für die Propagierung ihrer Pädagogik - und explizit als Gegenpol zum protestantisch-preußischen $\mathrm{Pu}$ blikationskartell und gegen die Macht des preußischen Zentralinstituts - gegründete „Deutsche Institut für wissenschaftliche Pädagogik“22 in Münster (mit Zweigstellen im Reich) (Müller, 2014; Tenorth, 2014)). Das bleibt bis in die Gegenwart des späten 20. Jahrhunderts - die zentrale Institution des Katholizismus, um auch in der Öffentlichkeit und in der Wissenschaft mit dem eigenen erziehungswissenschaftlichen und pädagogischpraktischen Standpunkt präsent zu sein. Spätestens hier, beim Blick auf die kommunikative Infrastruktur, wie sie die Verlage und Organisationen repräsentieren, sieht man auch unmittelbar die Kontinuitätslinien in die Zeit nach 1945: Der Herder-Verlag ist immer noch als katholischer Verlag präsent und als solcher der Öffentlichkeit bewusst, genauso wie Kösel oder Pustet oder Auer oder Schöningh. Auch die protestantische Seite verliert ihre publizistische Basis in der Pädagogik nicht, denn Beltz wie Quelle \& Meyer pflegten und pflegen z.T. auch diese Traditionen.

\footnotetext{
${ }^{20}$ Vgl. z.B. Einführung in die Sexualpädagogik.- Acht Vorträge im Zentralinstitut für Erziehung und Unterricht Berlin. Berlin (Mittler \& Sohn) 1921.

21 Joseph Schröteler (Hrsg.). im Auftrag der Zweigstelle Düsseldorf des Deutschen Instituts für wissenschaftliche Pädagogik und in Verbindung mit anderen Fachleuten: Die geschlechtliche Erziehung. Beiträge zur Grundlegung einer gesunden Sexualpädagogik. Düsseldorf 2. Aufl. 1929. und die Autoren stammen nur aus dem katholischen Milieu.

22 Dazu jetzt Markus Müller: Das Deutsche Institut für wissenschaftliche Pädagogik 1922-1980. Von der katholischen Pädagogik zur Pädagogik von Katholiken. (Veröffentlichungen der Kommission für Zeitgeschichte. Reihe B: Forschungen, Bd. 126), Paderborn: Schöningh 2014 sowie meine Rezension In: Theologische Literaturzeitung 139 (2014) 12, Sp. 1476-1478 und die Besprechung von Fritz Osterwalder in Bildungsgeschichte IHE 5(2015)1, S. 100-104.
} 
Die Stabilität der Konfessionszurechnung in der Konstruktion von Erziehungswissen bleibt also noch lange nach 1945 bestehen, wie es die noch in schöner Parallelität 1970/71 erschienenen konfessionell divergenten Lexika zeigen: Bei Herder erscheint das katholische "Lexikon der Pädagogik" und setzt damit die Herder-RoloffTradition der Zeit des endenden Kaiserreichs und den "Spieler" aus der Weimarer Republik fort $\left(.{ }^{23}\right.$ Beim evangelischen - Kreuz-Verlag in Stuttgart und Berlin gibt es jetzt - als Nachfolger von Schwarz - das „Neue Pädagogische Lexikon“24 (Groothoff und Stallmann, 1971), das (erstmals 1961 erschienen) die protestantische Tradition fortsetzt, beide in enger Kooperation mit Institutionen der jeweiligen Kirchen, hier mit dem deutschen Evangelischen Kirchentag. Solche Publikationen führen also in die Zeit nach 1945. Ich diskutiere hier zuerst die katholische Seite der Pädagogik in ihrer weiteren kommunikativen Infrastruktur und institutionellen Verankerung sowie in den Konsequenzen für den spezifischen Stil der Kommunikation über Erziehungsfragen.

\section{STABILITÄT UND WANDEL DES KATHOLISCHEN MiLIEUS: Gesellschaften, ZEITSCHRIFTEN,} KOMMUNIKATIONSPRAXIS NACH 1950

In seiner Analyse der Arbeit des Deutschen Instituts für wissenschaftliche Pädagogik bis zu seiner Auflösung 1980 hat Markus Müller diesen Prozess als Weg von der „Katholischen Pädagogik zur Pädagogik von Katholiken" stilisiert, freilich in eigener Ambivalenz. Er zeigt einerseits die Annäherung an die neuzeitlichen Wissenschaften und sogar - mit Doris Knab - an die empirische Bildungsforschung sowie, ebenfalls in der Person von Doris Knab, die Vernetzung mit dem alten protestantischgeisteswissenschaftlichen Milieu, das sich in der "Zeitschrift für Pädagogik" sein neues Organ geschaffen hat ${ }^{25}$ (sich freilich allmählich gegenüber den Gründern verselbständigt hatte) (Tenorth, 1986; Oelkers, 2004). Müller belegt andererseits aber auch die andauernden Konflikte mit dem Episko-

\footnotetext{
${ }^{23}$ Vgl. E.A. Roloff (Hrsg.), im Verein mit Fachmännern und unter besonderer Mitwirkung von Otto Willmann: Lexikon der Pädagogik. 5 Bde., Freiburg, Herder, 1913-1917. Jetzt: Lexikon der Pädagogik. Neue Ausgabe. Hrsg. Vom Willmann-Institut München/Wien; Ltg. der Herausgabe Heinrich Rombach. Freiburg/Basel/Wien 4 Bde., 1970/2. Aufl. 1971 - aber jetzt gibt es Überschneidungen bei Themenbetreuern, Karl Abraham, der Wirtschaftspädagoge, arbeitet hier wie bei Groothoff/Stallmann mit, Martin Stallmann selbst betreut die ev. Religionspädagogik, Albert Reble zählt zum Litt-Kreis (etc.)

${ }^{24}$ Hans-Hermann Groothoff und Martin Stallmann (Hrsg.): Neues Pädagogisches Lexikon. Stuttgart/Berlin (Kreuz-Verlag) 5. Aufl. 1971 Kreuz (Stuttgart) 1970 - und Überraschendes auch hier: Wolfgang Brezinka schreibt das Stichwort „Wandern und Reisen“, Karl Erlinghagen über „Kirche und Schule, kath.", Doris Knab (Berlin) über "Curriculumforschung“, - ansonsten findet sich die GWP von Klafki (und seinem Mitarbeiter Jürgen Diederich!) bis Mollenhauer, Roeder, Scheibe und Weniger, A. Flitner, Scheuerl, Theodor Schultze, Hans Thiersch oder Gerhard Wehle.

${ }^{25}$ Doris Knab - am Deutschen Institut von 1971-1982 Abteilungsleiterin - wird nach 1980 zugewählt, für den Wandel der Zeitschrift
} 
pat über die Möglichkeiten einer derart offen definierten katholischen Pädagogik und schließlich auch den allmählichen Bedeutungsverlust des Instituts in der Öffentlichkeit, nicht allein in der Öffentlichkeit der Kirche, sondern damit auch in der Wahrnehmung katholischer Pädagogik in der wissenschaftlichen und bildungspolitischen Öffentlichkeit.

Müller verweist in seinen Analysen zugleich auch auf den innerkirchlichen wissenschaftlichen Dissens über die Aufgabe des Instituts und die Möglichkeiten einer konfessionell geprägten Sozial- bzw. Erziehungswissenschaft. Der Münsteraner (später Würzburger) Kritiker der Knab-Position, Wilhelm Dreier (19281993), expliziter Vertreter einer christlichen Sozialwissenschaft, lehnt z.B. scharf die relative Autonomisierung gegenüber Amtskirche und Episkopat $a b$ und vertritt ganz offenkundig eine wissenschaftstheoretisch konservative Position. In wissenschaftstheoretischer Hinsicht war die Möglichkeit einer kirchlich-konfessionell gebundenen, hier also "katholischen“, Wissenschaft, konkret Erziehungswissenschaft dagegen - außerhalb des In- stituts - schon 1959 scharf attackiert worden. Das geschah in der Antrittsvorlesung, die Wolfgang Brezinka - katholisch! - 1959 zum Dienstantritt seiner Professur für Pädagogik an der - katholisch gebundenen - Pädagogischen Hochschule in Würzburg gehalten hatte ${ }^{26}$-zum Ärger seiner katholischen Zuhörer und des zuständigen Episkopats (Tenorth, 2004). Sie sehen nur die Differenz von Katholizität und Erziehungswissenschaft, aber nicht die von Brezinka hier und in der Folgezeit gleichzeitig bestärkte starke Verbindung von Konfessionalität und praktischer Pädagogik.

Wissenschaftstheoretisch hat Brezinka damit eine länger andauernde Diskussion ${ }^{27}$ angestoßen, in der er die strikte Differenz zwischen den Geltungsfragen von Wissenschaft und den - praktisch-normativen - Geltungsfragen von Erziehungswelten und den sie begründenden Weltanschauungen, Ideologien und Doktrinen, gleich welcher Provenienz, betont hatte. Hierin, in dieser wissensund wissenschaftstheoretischen $\mathrm{Ab}$ grenzung, ist Brezinka einer Position verwandt, die auch der als Erziehungswissenschaftler lehrende Je-

\footnotetext{
26 Wolfgang Brezinkas Antrittsvorlesung von 1959 hatte den Titel „Aufgaben und Probleme der Pädagogischen Hochschule in Bayern“. Seine Kritik galt dem Anspruch, "konfessionelle Wissenschaft", also auch eine "katholische Erziehungswissenschaft", begründen zu können;

27 Prominent geworden ist seine Kritik an konfessionell gebundenen Lehrbüchern (von Hubert Henz bis Fritz März) und die anschließende Debatte mit Heinrich Rombach, dem Herausgeber des Herder-Lexikons Pädagogik, der auch - als Philosoph, der von Haus aus ist, - Brezinka wissenschaftstheoretisch zu entkräften suchte, vgl.W.B: Die Krise der wissenschaftlichen Pädagogik im Spiegel neuer Lehrbücher. In: Zeitschrift für Pädagogik 12 (1966), S. 53-88 und gegen Rombach W.B..: Über den Wissenschaftsbegriff der Erziehungswissenschaft und die Einwände der weltanschaulichen Pädagogik. In: Zeitschrift für Pädagogik 13 (1967), S. 135-168; dagegen kritisch Heinrich Rombach: Der Kampf der Richtungen in der Wissenschaft (Eine wissenschaftstheoretische Auseinandersetzung). In: Zeitschrift für Pädagogik 13 (1967), S. 37-69; die breite Anschlussdiskussion ist u.a. dokumentiert bei Wilhelm Büttemeyer (Hrsg.): Der Positivismusstreit in der deutschen Erziehungswissenschaft. München 1976.
} 
suit Karl Erlinghagen ebenfalls eingenommen hatte. Der konnte, wie Brezinka, den Erkenntnisgewinn einer autonomen Wissenschaft anerkennen und nutzen und zugleich - normativ dann in katholischen Traditionen verankert - in praktischen Erziehungsfragen z.B. die Notwendigkeit einer religiös gebundenen Erziehung propagieren, dabei auch das deutsche Staatskirchenrecht verteidigen und eine laizistische Erziehung scharf ablehnen. Das alte Programm hätte sich damit wissenschaftstheoretisch, programmatisch und in der Forschung erledigt, politisch-weltanschaulich aber erhalten, wie manche Kontinuitätsaspekte zeigen. Auch der Jesuitenorden lässt sich ja seine Hochschulen staatlich bezahlen, so wie die katholische Kirche in Bayern ihre Konkordatslehrstühle, die es ja auch in der Pädagogik gibt, nicht nur in der Geschichte, Philosophie oder Soziologie.

Im katholischen Milieu wird diese Differenz von theoretischer und praktischer Geltung aber keineswegs generell so gesehen (und auch im Kontext politisch engagierter Erziehungswissenschaft nicht, die schon Brezinkas Position entschieden attackierte - von dieser Fraktion wird noch die Rede sein). Nach wie vor gibt es das Programm einer im Glauben, d.h. konfessionell und d.h. christlich oder ka- tholisch geprägten Wissenschaft, und nicht nur für die Erziehungswissenschaft. Signifikant dafür ist für die katholische Seite v.a. die Position und Praxis der Görres-Gesellschaft. ${ }^{28}$ Sie ist relevant für die Wissenschaften und die Wissenschaftspolitik generell und natürlich auch für die Pädagogik wie für die anderen ideologiebrisanten Geisteswissenschaften, aber auch verantwortlich für das "Staatslexikon" (dessen protestantisches Gegenstück natürlich auch nicht fehlt Kunst und Grundmann, 1966). Die Görres-Gesellschaft versteht sich heute "christlich", und zwar, so die aktuelle Selbstbeschreibung, auf der Basis des „Bekenntnisses zum christlichen Glauben und Menschenbild in der Tradition der Katholizität, zur Freiheit als personaler und sozialer Kategorie der Staats- und Gesellschaftsgestaltung". Sie rekrutiert ihre Mitglieder katholisch, organisiert für ihre Mitglieder Tagungen, verleiht Stipendien und ediert Zeitschriften, erziehungswissenschaftlich bis heute aktuell die "Vierteljahrsschrift für wissenschaftliche Pädagogik". Dabei hat sie sich - grundsätzlich - zum "Ziel gesetzt, in Bewahrung ihres im katholischen Glauben wurzelnden Gründungsauftrages wissenschaftliches Leben auf den verschiedenen Fachgebieten anzuregen und zu fördern und die

\footnotetext{
${ }^{28}$ Basal kennt ihre Geschichte schon wikipedia: „Die Görres-Gesellschaft wurde am 25. Januar 1876 von katholischen Gelehrten und Publizisten in Koblenz unter der Bezeichnung Görres-Gesellschaft zur Pflege der katholischen Wissenschaften gegründet. Mitbegründer und Hauptinitiator war der spätere bayerische Ministerpräsident und deutsche Reichskanzler Georg von Hertling, der auch erster Präsident der Görres-Gesellschaft wurde."

29 Selbstbeschreibung Web-Site, 29.08.2015: „Die „Görres-Gesellschaft zur Pflege der Wissenschaft“ .... dient dem Zusammenschluss aller wissenschaftlich Interessierten, deren Denken und Forschen die verpflichtende Bedeutung der christlichen Tradition anerkennt. Das wissenschaftliche Weltbild,
} 
Gelegenheit zum interdisziplinären Austausch zu bieten. "29 Zielsetzung wie Praxis entsprechen also dem Normalmodell einer Gemeinschaft von Wissenschaftlern, die ihre eigenen Interessen und ihre genuinen theoretischen Programme durch Verbandsaktivitäten forcieren und öffentlich vertreten wollen. In ihrer Geschichte $(\text { Morsey, 1986) })^{30}$ bildete die Pädagogik zwar erst spät eine eigene Sektion, aber sie zeigt, wie sich katholische Pädagogik heute stilisiert, um in den Wissenschaften kommunikationsfähig zu bleiben - und das ist dann doch überraschend, wie sich am Selbstverständnis und der publizistischen Pra- xis der pädagogischen Zeitschrift der Görres-Gesellschaft belegen lässt.

Diese Zeitschrift, die Vierteljahrsschrift, schon vor 1945 gegründet, heute im 91. Jahrgang, war schon immer argumentativ ein Organ der Erziehungsphilosophie, in der operativen Pädagogik eher traditional, nah bei den Herbartianern, vom katholischen Deutschen Institut für wissenschaftliche Pädagogik gegründet und in den ersten Herausgebern eindeutig kirchlich bestimmt (Müller, 2014) Nach 1945 zunächst von Alfred Petzelt und seiner (neukantianischen) Schule geprägt, aber auch bestimmt von dessen katholischen

das die Neuzeit beherrscht hat, befindet sich in einer Krise; sowohl die naturwissenschaftlichen wie die geisteswissenschaftlichen Disziplinen erleben einen Umwandlungsprozess, in dem neue Möglichkeiten der Erkenntnis sich abzeichnen. ... Die der Wissenschaft gesetzten Aufgaben sollen hier den modernen Anforderungen entsprechend auf christlichem Boden erfüllt werden. ..."

${ }^{30}$ Zur Geschichte vgl. Rudolf Morsey: Görres-Gesellschaft. In: Görres-Gesellschaft (Hrsg.), Staatslexikon — Recht Wirtschaft Gesellschaft, 2.Bd., 7. Aufl. 1986, Freiburg im Breisgau]. Die website nennt die relevanten Kontexte und Funktionen: „Unbeschadet ihrer Gründung als eine Art von Notund Verteidigungsgemeinschaft ... durch den Abbruch des Kulturkampfs erleichtert ... Aktivität als „Laienorganisation“ von Gelehrten ... ohne eigene Theologische Sektion und ohne Konkurrenz zum neu gestärkten kirchlichen Lehramt ... geistige Auseinandersetzung mit den Problemen der modernen Welt ... auf dem Fundament des „Bekenntnisses zum christlichen Glauben und Menschenbild in der Tradition der Katholizität, zur Freiheit als personaler und sozialer Kategorie der Staats- und Gesellschaftsgestaltung“ sowie aus der Überzeugung, daß der Verdacht einer Unvereinbarkeit von wissenschaftlicher Rationalität und christlichem Glauben überholt ist (L. Boehm). Die G.-G. begann ... ihre Arbeit in vier Fachsektionen: für Rechts- und Sozialwissenschaft (seit 1877), Philosophie (seit 1877), Geschichte (seit 1878) und Naturwissenschaften (seit 1906). ... gegen staatliche Omnikompetenz konzipierte "Staatslexikon“ (1. Auflage 1889-1897; 6. Auflage 1951-1971) ... Zu den rasch begründeten Zeitschriften der G.-G., die bis heute erscheinen, ... "Historische Jahrbuch“ (seit 1880), die „Römische Quartalschrift für christliche Altertumskunde und Kirchengeschichte“ (seit 1887), das "Philosophische Jahrbuch“ (seit 1888), ... inzwischen zahlreiche weitere ... Errichtung neuer Sektionen ... Kirchenmusik, Pädagogik, Psychologie und Psychotherapie ... von vorneherein ein „interdisziplinäres Element ... Beitrag zur wissenschaftlichen Pluralität. ... Ihrer Zielsetzung entsprechend war die G.-G. - die vor dem 1. Weltkrieg an der römischen Kurie zu Unrecht in den Verdacht „modernistischer Häresie“ geraten war - nach 1933 den nationalsozialistischen Machthabern verhasst. ... zunächst noch - insbesondere wegen ihrer Auslandsinstitute und des internationalen Ansehens ihres Präsidenten Finke - geduldet, allerdings in ihrer Arbeit erheblich behindert, am 11. Juni 1941 dann vom Reichsminister des Innern aufgelöst und ihr Vermögen beschlagnahmt."

${ }^{31} \mathrm{Zu}$ Petzelt u.a. Peter Vogel: Die neukantianische Pädagogik und die Erfahrungswissenschaften vom Menschen. In: J. Oelkers, W.K. Schulz und H.-E. Tenorth (1989,Hrsg. ):Neukantianismus. Kulturtheorie, Pädagogik und Philosophie. Weinheim, Basel, S. 127-164, bes. S. 152ff. 
Grundüberzeugungen ${ }^{31}$, wird sie seit 1969 „in Verbindung mit“, dann „Im Auftrag der Görres-Gesellschaft herausgegeben", aktuell von Winfried Böhm, Ursula Frost (geschäftsführend), Volker Ladenthin, Gerhard Mertens. In der Redaktion arbeiten noch Matthias Burchardt und Dr. Markus Rieger-Ladich mit, beide nicht ganz so eindeutig in ihrer Theoriepraxis konfessionell-katholisch zurechenbar. Das Programm lautet, jetzt anscheinend ganz ohne Bezug auf die katholische Referenz: ${ }^{32}$

„Die Zeitschrift ... bietet Einblick in aktuelle wissenschaftliche Diskussionen in der ganzen Breite des Faches, wobei der Schwerpunkt in einer philosophischen und zeitdiagnostischen Reflexion besteht. Pädagogik als Wissenschaft und als gesellschaftliche Praxis soll hier in einer Weise zur Sprache kommen, die die Sensibilität in der Wahrnehmung von Gegenwartsphänomenen schärft und den Umgang mit ihnen verantworten hilft. ... bildet die Zeitschrift ein Forum, das eine Auseinandersetzung mit den gesellschaftlich-kulturellen Bedingungsgefügen von Erziehung und Bildung und eine Analyse ihrer Schauplätze ebenso ermöglicht wie eine Klärung pluraler wissenschaftlicher Zugangsweisen und eine Stärkung pädagogisch reflektierter Positionen, die eine Perspektive der Verantwortung eröffnen."

Das liest sich nun keineswegs typisch katholisch, selbst die Kategorie der "Verantwortung" findet sich andernorts, sogar in der Bildungsreflexion von Max Horkheimer. Auch die Basisprämisse der Görres-Gesellschaft „Das wissenschaftliche Weltbild, das die Neuzeit beherrscht hat, befindet sich in einer Krise" - stiftet nicht sofort eine kirchlich-katholische Identität, "Zeitdiagnose“ scheint eher die zentrale Intention. Das bestätigt auch ein eigenes „Handbuch der Erziehungswissenschaft" (Mertens, Frost, Böhm, Koch und Ladenthin, 2007), das aus diesem Kreis ediert wurde, ausdrücklich gegen sozialwissenschaftliche "Modeerscheinungen" inszeniert. Schon in der Frage nach der "Ein-

\footnotetext{
32 Auszüge aus der Selbstbeschreibung, website Vierteljahrsschrift für wissenschaftliche Pädagogik, eingesehen 2015, vollständig: „Die Zeitschrift wendet sich an alle, die pädagogische Themen aus Theorie und Praxis reflektieren möchten. Sie bietet Einblick in aktuelle wissenschaftliche Diskussionen in der ganzen Breite des Faches, wobei der Schwerpunkt in einer philosophischen und zeitdiagnostischen Reflexion besteht. Pädagogik als Wissenschaft und als gesellschaftliche Praxis soll hier in einer Weise zur Sprache kommen, die die Sensibilität in der Wahrnehmung von Gegenwartsphänomenen schärft und den Umgang mit ihnen verantworten hilft. Erziehungs- und Bildungsprozesse in unterschiedlichen Kontexten als solche zu beschreiben und zu unterstützen, ist keineswegs mehr eine klar zu bestimmende Aufgabe. Einerseits werden durch Politik und Ökonomie, Medien und verschiedenste gesellschaftliche Gruppen zunehmend Realitäten geschaffen, die die Prozesse unabsehbar bestimmen und verändern, so daß pädagogische Intentionen fragwürdig werden. Andererseits erfolgen die Beschreibungen und Aufgabenformulierungen innerhalb der Pädagogik bzw. Erziehungswissenschaft zunehmend getrennt und verschieden. In dieser Situation bildet die Zeitschrift ein Forum, das eine Auseinandersetzung mit den gesellschaftlich-kulturellen Bedingungsgefügen von Erziehung und Bildung und eine Analyse ihrer Schauplätze ebenso ermöglicht wie eine Klärung pluraler wissenschaftlicher Zugangsweisen und eine Stärkung pädagogisch reflektierter Positionen, die eine Perspektive der Verantwortung eröffnen."
} 
heit" des Pädagogischen gegen die „Heterogenität" des aktuellen Diskurses, wie sie für die "Moderne im Umbruch" charakteristisch sei, ist das Unternehmen leicht als Projekt der Bildungs- und Erziehungsphilosophie identifizierbar. Die sieht sich auch generell angesichts der Karriere empirischer Bildungsforschung im Abseits versucht und neue Anstrengungen, die jetzt „in der Tiefe des jüdischchristlich geprägten abendländischen Denkens verwurzelt" sein sollen (Bd. 1, S. XII). das nähert sich der katholischen Tradition, verstärkt auch durch die, natürlich möchte man sagen, intensiv reklamierte "präskriptive Dimension" (S. XIII), die hier für grundlegend erklärt wird. Auch in dieser Lagebeschreibung wie in der "zeitdiagnostischen“ Absicht erinnern die programmatischen Sätze zum eigenen Selbstverständnis und in der Lagebeschreibung der Erziehung in Theorie und Praxis deshalb auch bald eher an Traditionen der kritischen Erziehungswissenschaft und insbesondere der „Kritischen“ Theorie in der Pädagogik. ${ }^{33}$ Insgesamt spiegelt sich in dieser Programmatik also eine neue Fraktionierung innerhalb der Erziehungswissenschaft, die primär von Argumentformen bestimmt scheint, aber in einer eigenartigen Weise den alten Traditionalismus des konfessionell geprägten Abwehrkampfes gegen die Moderne mit Argumentationsformen und Gruppierungen verbindet, die früher jeweils selbst zur Gegenseite gerechnet wurden, nämlich mit politischen Gruppierungen, die sich selbst auf der linken Seite des ideologischen und politischen Spektrums platzieren und platziert werden. Man darf, so würde ich es zuspitzen, auf der katholischen Seite der erziehungswissenschaftlichen Tradition eine offene Politisierung des konfessionellen Standpunktes erkennen, zeitdiagnostisch diagnostizieren, um in der Argumentation zu bleiben.

Solche - in der historischen Dimension dann doch überraschende Bündnisse - zeigen sich in vielen aktuellen Themen, die hier behandelt werden, u.a. in der Opposition gegen „Bologna“, also Trends der Hochschulund Universitätspolitik, in der Kritik an Bildungsstandards (Ladenthin), in Abgrenzungspraktiken gegenüber der Bildungspolitik und in eigener Bündnispolitik z.B. mit der GEW oder mit linker Zeitdiagnostik (Frost, 2006). Das soziale Netzwerk der aktuellen Herausgeber zeigt aber, dass es hier Grenzen nach links gibt, allerdings gleichzeitig ein breites Bündnis in der Erziehungsphilosophie: Winfried Böhm, selbst Heitger-Schüler seit seinem Studium in Bamberg ${ }^{34}$, verweist bereits auf das Salzburger Symposion, das dort seit mehr als 50 Jahren

\footnotetext{
33 Andreas Gruschkas Zeitschrift „Pädagogische Korrespondenz“(vom „Institut für Pädagogik und Gesellschaft" herausgegeben) versteht sich - in der Nachfolge der Theorie Adornos - als "Zeitschrift für kritische Zeitdiagnostik in Pädagogik und Gesellschaft“, ausgehend von „Kältestudien“ (dazu meinen Kommentar HET: Adorno, das Wetter und wir. In: Pädagogische Korrespondenz H.7, 1990, S. $41-49)$.

${ }^{34}$ Böhm ist nur zufällig in Würzburg gelandet und bei Albert Reble promoviert, weil Marian Heitger, sein Erstbetreuer, in Bamberg, an einer PH, nicht promovieren konnte.
} 
veranstaltet wird, transzendentalkritisch, vereint zwischen den beiden Fraktionen, die diese erziehungsphilosophische Theorieschule zwischen Marian Heitger einerseits, Wolfgang Fischer und Jörg Ruhloff andererseits aufweist. Beide sind aber nah an der, jetzt wieder katholischen, im Detail franziskanischen, von Petzelt inspirierten Tradition des Neukantianismus zu platzieren. Salzburg wiederum lädt auch andere Gäste ein, früh schon aus der geisteswissenschaftlichen Tradition, auch dann aber in kritischer wissenschaftstheoretischer Wendung und schon immer "zeitdiagnostisch", jedenfalls in der Argumentation philosophisch und grundlagentheoretisch (Kauder, 1925).

Die Herausgeber belegen zugleich dennoch, dass es weiterhin auch ,katholische" Kontakte gibt, international, wie sie Böhm nach Frankreich (Michel Soëtard), Italien, Argentinien oder Polen unterhält, wobei dann auch die theoretischen Differenzen zum linken erziehungsphilosophischen Lager deutlicher sichtbar werden. Sein theoretischer Grundbegriff, der der „Person", die als Referenz in Karol Woytila auch einen veritablen Papst hat (Johannes Paul II), findet sich in anderen Fraktionen der erziehungs- und bildungsphilosophischen Tradition in dieser Weise nicht, obwohl auch sie von "Person" reden (Eykmann und Böhm, 2006). ${ }^{35}$ Die Netzwerke funktionieren aber nicht nur theoriestrategisch, sondern auch ganz pragmatisch, berufungspolitisch z.B., wie sich bei einer anderen Herausgeberin, $U r$ sula Frost, zeigt. Sie ist Professorin in Köln ${ }^{36}$, dort lehrt auch, in der Philosophie, der Leiter der "Sektion Pädagogik" der Görres-Gesellschaft Prof. Dr. Michael Obermaier, zusammen mit Professor Dr. Gerhard Mertens, einem Herausgeber des "Handbuchs"; Frau Rita Molzberger, ebenfalls in der GörresGesellschaft leitend aktiv, gehört zum universitären Personal von Frau Frost (etc.).

Versucht man ein Fazit, dann kann man für den theoretischen und reflexiven Kontext der katholischen Pädagogik sagen: Ihren frühen und folgenreichen Ausdruck findet sie zuerst - immer noch als katholische Pädagogik verstanden - in Otto Willmanns Idee einer paedagogia perennis, die sich aristotelisch-thomistisch begründet (als Argumentfigur im katholischen Milieu nie ganz aufgegeben $^{37}$ ) und die in der Katholizität die "Vollendung des Menschentums" sieht. Aktuell ist sie aber erziehungsphilosophisch, allerdings mit eindeutigem Profil, dann aber auch in politisch überraschenden Bündnissen, thematisch sogar offen nach links. In-

\footnotetext{
35 Dazu die Beiträge in W. Eykmann/W. Böhm (Hrsg.): Die Person als Maß von Politik und Pädagogik. Würzburg 2006. Der Band zeigt, dass Böhms katholisch begründeter Personbegriff deutlich unterschieden ist von einer sozialwissenschaftlichen Argumentation z.B. bei N. Luhmann/K.E. Schorr (Hrsg.): Zwischen Absicht und Person. Fragen an die Pädagogik. Frankfurt a.M. 1992.

36 „Lehrgebiete“, lt. Website: „Historisch-systematische Pädagogik, philosophische Grundlegung der Pädagogik, insbesondere Bildungs- und Erziehungstheorie, interdisziplinäre Aspekte zwischen Theologie und Pädagogik".

37 Böhm erwähnt das Thema, bei Hubert Henz: Lehrbuch der systematischen Pädagogik. Freiburg 1964 regiert er noch, Otto Willmann gab ihm zuletzt theoretische Kontur.
} 
stitutionell ist sie in den Filiationen des deutschen Universitätssystems abgesichert, verstärkt auch dank der Prämissen des bayerischen Konkordats mit dem Vatikan von 1924 (das mit der Abschaffung der Konfessionsschulen nach 1967/68 dann 1974 bestätigt wurde), auf dessen Grundlage bis heute in Bayern mindestens ein Lehrstuhl für allgemeine Pädagogik (und für Soziologie und mittelalterliche Geschichte und für Philosophie) an jeder bayerischen Universität nach Konfessionskriterien katholisch besetzt wird und nur mit dem notwendigen "nil obstat" des jeweiligen Landesbischofs in Bamberg oder München, Regensburg, Bayreuth oder Würzburg besetzt werden darf. ${ }^{38}$ Konkordate sichern aber auch die Katholisch-Theologischen Hochschulen, damit auch deren Religionspädagogik, aus der vor 1945 und dann nach 1945 die folgenreiche eigene Systematik katholischer Pädagogik wie die von Göttler kam. Aber sowohl innerhalb der katholischen "christlichen Sozialwissenschaft" von Wilhelm Dreier, als auch beim Episkopat ist eine selbstbewusst-autonome Form von Wissenschaft, wie sie z.B. nach 1970 im Kontext der Bildungsreform im Deutschen Institut entwickelt wird, schon bald wenig geachtet, sie gilt als $\mathrm{zu}$ distanziert, und zugleich in Bezug auf die erwarteten Leistungen von Wissenschaft zwischen unmittelbarer Handlungsorientierung und Normbe- gründung nicht den kirchlichen und konfessionellen Erwartungen entsprechend. 1980 findet diese Praxis einer auch extern anerkannten Wissenschaft an den internen Querelen im Katholizismus ein Ende, jetzt gilt institutionell und kirchlich eher wieder "Wagenburg-Mentalität", allenfalls in der Görres-Gesellschaft relative Offenheit in die gesamte erziehungswissenschaftliche Diskussion hinein, wenn man - anders als vor 1993 bei Dolch und Fischer - die empirische Bildungsforschung ausnimmt.

\section{Protestantismus IN DER PÄDAgOGIK NACH 1950: MotoR DES FORTSCHRITTS}

Institutionell natürlich abgesichert wie die katholische, also in Lehrerseminaren, Pädagogischen Hochschulen und theologischen Fakultäten, damit auch in der Religionspädagogik oder praktischen Theologie, theoretisch aber eher sektenspezifisch ausgefächert zwischen ,"christlicher Pädagogik " und strenger Kirchenbindung, ist die einschlägig evangelische Theoriefraktion der Erziehungswissenschaft heute eher implizit protestantisch. ${ }^{39}$ Das galt im disziplinären Selbstverständnis schon für die preußische, staatsnahe, geisteswissenschaftliche, sich selbst als "christlich" verstehende Pädagogik in der Nohl-FlitnerTradition. Das gilt heute systematisch

\footnotetext{
${ }^{38}$ Berufungen zeigen dann die Netzwerke, die man z.B. auf der Rheinschiene von Koblenz über Köln nach Neuss und Bonn oder Duisburg verfolgen kann, auch in Bayreuth (bei Lutz Koch) oder in Würzburg, wo Duisburg und Böhm sich arrangieren, wenn Dörpinghaus berufen wird (etc.).

${ }^{39}$ Einschlägige Thematisierungen von Religion zeigen das, wie sie sich von Hentig bis Oelkers oder Benner finden.
} 
vor allem in der theoretischen Grundbegrifflichkeit, die frei von aller Kirchlichkeit rein säkular vorliegt, aber implizit doch christlich imprägniert ist. Dieses quasi-neutrale, nicht offen kirchlich gebundene Selbstverständnis wird institutionenpolitisch natürlich durch das andere Kirchenverständnis im Protestantismus gestützt, es findet aber auch erziehungswissenschaftlich seine eigene theoretische Begründung. Man kann sie vor allem im Verständnis der Bildungstheorie suchen, wie sie in den Theorietraditionen vorliegt, die ich dem Protestantismus zurechne und die sich selbst, im Konfliktfall, auch so outen.

Zwischen dem Funktionsprimat von „Bildung“ - klassisch ja die Selbstbildung des Subjekts in einem freien, autonomen Weltzugang - und dem kirchlich erwarteten „Bekenntnis“ besteht nämlich für die protestantisch infizierte Bildungstheorie und Religionspädagogik kein Spannungsverhältnis und schon gar kein Widerspruch, wie er für die Bekenntnistheologie des Katholizismus von den Protestanten durchaus unterstellt wird. In diesem Verständnis protestantischer Religiosität werden die theoretischen
Implikationen der Innerlichkeitspädagogik, die pikanterweise aus dem französischen Jansenismus geborgt sind ${ }^{40}$, und die praktischen Implikationen der Gleichsetzung von Bildung und Bekenntnis aber überhaupt nicht mehr diskutiert, sondern positiv, gelegentlich auch nur implizit, reklamiert (vgl. Tenorth, 1997, 1999, 2012). ${ }^{41} \mathrm{Ge}-$ sellschaftsgeschichtlich und -politisch wird auch die Funktion von Bildung als säkulare „Bildungsreligion“42 damit weiter bekräftigt, auch in dem Glauben, Bildung und Bekenntnis, Wissenschaft und praktische Normierung von Erziehungsverhältnissen ließen sich im Protestantismus und seinem Freiheitspathos in ihrer Funktionslogik harmonisch vereinbaren wie man von Nipkow, Dreesen und Schweitzer bis zu Hentig hören kann, also bei religionspädagogischen Experten und gläubigen Laien, im Allgemeinen also.

Auch der Protestantismus ist natürlich nicht nur theoretisch und in Einzelpersonen, sondern in eigenen kommunikativen Organisationen und Netzwerken öffentlich folgenreich präsent. Die kommunikativ relevante Organisation ist hier - ne-

\footnotetext{
40 So Fritz Osterwalder.

${ }^{41}$ Belege dafür liefert meine Kontroverse mit Karl-Ernst Nipkow und Schieder, vgl. mit den Verweisen auf die Texte der Opponenten - Heinz-Elmar Tenorth: Reform - Pädagogik - Religion. In: Der Evangelische Erzieher 49(1997), S. 376-384.; ders.: Schule - Religion - Zivilreligion. Zur weiteren Problematisierung eines jetzt schon schwierigen Verhältnisses. In: Ch.Th. Scheilke und F. Schweitzer (1999, Hrsg.): Religion, Ethik, Schule. Bildungspolitische Perspektiven in der pluralen Gesellschaft. Münster (usw.)., S. 175-186, ders.: Bildung und Konfessionalität. Zur Geschichte einer deutschen Tradition oder: vom Bedeutungsverlust einer historisch bedeutsamen Struktur. In: D. Owetschkin (2012, Hrsg.): Tradierungsprozesse im Wandel der Moderne. Religion und Familie im Spannungsfeld von Konfessionalität und Pluralisierung. Klartext, Essen, . 75-87.

${ }^{42}$ Historiker nutzen diesen Begriff, um die Funktion von „Bildung“ im deutschen Kontext und für das Bildungsbürgertum zu charakterisieren. In kritischer Nutzung jüngst z.B. Hans Pechar: Humboldt in der Massenuniversität? Vom Elend der neuhumanistischen Bildungsreligion. In: Brigitte Kossek und Charlotte Zwiauer (2012, Hrsg.): Universität in Zeiten von Bologna. Wien, S. 69-82.
} 
ben der Tradition, die sich in der geisteswissenschaftlichen Pädagogik seit 1918/20, in der Nohl-WenigerSchule an Universitäten und Pädagogischen Hochschulen im nördlichen, protestantischen Deutschland nach 1945/50 und in der "Zeitschrift für Pädagogik" ihre Einflusschancen dauerhaft gesichert hat - bis heute das Comenius-Institut, die „Evangelische Arbeitsstätte für Erziehungswissenschaft" (Elsenbast, Pithan, Schreiner und Schweitzer (Hrsg.) 2004, $)^{43}$, wie sie sich heute selbst beschreibt. Mich interessiert hier aber nicht primär das programmatische Selbstverständnis und die aktuelle Politik, die erwartbar im Jargon von "Dialog", „Interdisziplinarität" und Verantwortung beschrieben wird, aber immerhin, anders als bei den Katholiken, explizit "aus evangelischer Verantwortung" und auch in "evangelischer Bildungsverantwortung" 44, ohne die Rhetorik des ,,jüdisch-christlichabendländischen" Horizonts, von der die eindeutige Kirchlichkeit ja nur verdeckt wird.
Mich interessiert hier nur die disziplinäre Rolle des Instituts für die Veränderung der Erziehungswissenschaft nach 1960. Nur am Rande kann ich deshalb erinnern, dass sich auch Wilhelm Flitners folgenreiche Diskussion über „Das Selbstverständnis der Erziehungswissenschaft in der Gegenwart" dem Institut (Flitner, 1956), und zwar der ersten Tagung des Instituts über "Grundfragen der Erziehungswissenschaft" vom 5. bis 8 . März 1956 in Arnoldshain verdankt und als Band 1 der „Pädagogischen Forschungen" publiziert wurde. Auf dieser Tagung redete ebenfalls Hans Mieskes (aus der Jenaer WinnefeldSchule der pädagogischen Tatsachenforschung) über "Auftrag und Gegenstand der empirischen Forschung in der Pädagogik von heute", das basale Thema war das Verhältnis von Theologie und Pädagogik, die also hermeneutisch-pragmatisch und in empirischer Methodik definiert wird. Carl Ludwig Furck, der Mentor der Sache, berichtet davon wiederum unter der Überschrift „Erziehungswissen-

\footnotetext{
$43 \mathrm{Zu}$ seiner Geschichte umfassend Volker Elsenbast, Annebelle Pithan, Peter Schreiner und Friedrich Schweitzer (2004, Hrsg.): Wissen klären Bildung stärken. 50 Jahre Comenius Institut. Münster, mit einem ausführlichen Abschnitt über „Erziehungswissenschaft und evangelische Bildungsverantwortung" und analytisch relevanten Beiträgen u.a. von Carl-Ludwig Furck (Die ersten Jahre, 1954-1970. S. 17-45), Nipkow und Schweitzer sowie einem kompletten Verzeichnis der Veröffentlichungen.

44 Dafür reicht die Lektüre der website, wo sich u.a. die folgenden Sätze finden (26.09.2015): Zur Gründung: „Das Comenius-Institut wurde 1954 von evangelischen Lehrer- und Erzieherverbänden in Verbindung mit der EKD gegründet als eingetragener Verein mit Sitz in Münster/Westfalen“, Zur Arbeit: „Unser Auftrag ... Forschung und Entwicklung ... Als „Evangelische Arbeitsstätte für Erziehungswissenschaft e.V." dient das Comenius-Institut der Förderung von theoretischen Erkenntnissen und praktischen Lösungen gegenwärtiger Bildungs- und Erziehungsprobleme in Kirche, Schule und Gesellschaft aus evangelischer Verantwortung. ... Das Comenius-Institut bearbeitet Aufgaben im Spannungsfeld von Pädagogik und Theologie, Wissenschaft und Öffentlichkeit, Praxis und Politik, Kirche und Schule. ... Es hat die Funktion, Grundfragen evangelischer Bildungsverantwortung zu klären, Grundlagen für den Dialog zwischen unterschiedlichen Gruppen und Interessenten im Bildungsbereich bereitzustellen, zu beraten, zu koordinieren und zu vernetzen."
} 
schaft, Theologie und Soziologie" und für die weitere Arbeit hebt er besonders hervor, dass die Tagungen des Instituts eine wichtige Gelegenheit für den wissenschaftlichen Nachwuchs waren, sich kennenzulernen und frei über ihre Arbeit zu kommunizieren (S. 27f.). Das geschah dann auch. Ein erstes wichtiges Thema hieß 1957: „Formen gültiger erziehungswissenschaftlicher Aussagen“ (ebd., S. 29), dann ging es wiederholt um die „Theorie der Schule"; Wolfgang Kramp (19271983) hat hier theoretisch und wissenschaftstheoretisch auch gelernt, bevor er seinen zäsursetzenden Artikel zu diesem Thema schrieb (Kramp, 1970). Alles fand immer in Hofgeismar statt, für die Protestanten ein genauso wichtiger Ort wie für die Jungsozialisten, wie ich doch hervorheben will. 1960 wurde dann die "Studienkommission Pädagogik und Soziologie“" gegrün$\operatorname{det}$ (S. 30ff.).

Über die Rolle des ComeniusInstituts kann man also historisch schon diskutieren (Zeitgenossen haben das auch getan ${ }^{45}$ ), auch methodisch kontrolliert und kann seine Arbeit deshalb auch begründet für bedeutsam erklären. Meine These heißt denn auch, dass sich dieses protestantische Institut mit seinen Aktionen und Publikationen als Motor der Erneuerung der Erziehungswissenschaft, nicht der Abwehr, wie das die katholische Seite in der Bundesrepublik wahrgenommen und ge- wirkt hat. Mein einziger ausführlich genutzter Beleg an dieser Stelle sollen - schon aus Umfangsgründen die zehn Bände zum Thema „Gesellschaft und Erziehung“ darstellen, die innerhalb der „Pädagogischen Forschungen“ erschienen sind. Sie stellen insgesamt als "Veröffentlichungen des Comenius-Instituts" die Teilreihe „Erziehungswissenschaftliche Studien“ dar, wo auch, in denen auch, um die „Kluft“ zwischen deutscher und amerikanischer Soziologie zu schließen (Furck, ebd., S. 31), z.B. Orville G. Brims "Soziologie des Erziehungswesen" von Peter Roeder übersetzt erschienen ist.

Die Reihenherausgeber insgesamt waren Carl-Ludwig Furck (1923-2011), Nohl-Schüler, dann in Berlin und Hamburg lehrend und forschend sowie bildungspolitisch-reformerisch im Umkreis der Sozialdemokratie zuerst in Berlin aktiv, Mitgründer der Deutschen Gesellschaft für Erziehungswissenschaft (Berg, Herrlitz und Horn, 2004), Mitherausgeber der Zeitschrift für Pädagogik seit 1966. $\mathrm{Zu}$ den Reihenherausgebern gehörte ferner Dietrich Goldschmidt (19141998), als „Halbjude“ von den Nazis verfolgt, Direktor am Max-PlanckInstitut für Bildungsforschung, Ingenieur von Beruf, aber in Soziologie bei Helmut Plessner in Göttingen promoviert, in seinen Forschungen eher Soziologe als Erziehungswissenschaftler, sowie die Religions-

\footnotetext{
45 Vgl. Hans Bernhard Kaufmann u.a. (Hrsg.): Kontinuität und Traditionsbrüche in der Pädagogik. Ein Gespräch zwischen den Generationen. Weinheim/Basel 1991 - und Kaufmann, Direktor des Comenius-Instituts, betont in seinem Vorwort noch einmal den Konsens zwischen den Disziplinen, wie ihn der Bildungsbegriff und die gemeinsame „Bildungsverantwortung" stiften.

${ }^{46}$ Dazu im Einzelnen Dietlind Fischer: Ingeborg Röbbelen (1923-1970), Die erste Direktorin des
} 
pädagogin Ingeborg Röbbelen ${ }^{46}$ (19231970). Sie war die erste Leiterin des Comenius-Instituts (seit 1956 und bis 1970), auch in Göttingen - in der Theologie - promoviert. Man findet also ein Göttinger Netz, protestantisch und geisteswissenschaftlich, soziologisch und theologisch sozialisiert und durch enge Beziehungen und gemeinsame Interessen verbunden, nahezu ein Leben lang.

Die Autoren der Reihe wiederum belegen einerseits die interdisziplinäre Rekrutierung, zum anderen die auch von Furck schon herausgestellte folgenreiche Funktion für den wissenschaftlichen Nachwuchs, für die Erziehungswissenschaft schließlich die zentrale Rolle sowohl im Allgemeine als auch bereichsspezifisch.

Das allgemeine Selbstverständnis, ja die markante Zäsur formuliert und proklamiert der Titel von Band 1: „Erziehungswissenschaft als Gesellschaftswissenschaft". Er ist 1969 erschienen, herausgegeben vom Reihenherausgeber Goldschmidt zusammen mit Christa Händle, damals schon im Max-Planck-Institut für Bildungsforschung, Mario Rainer Lepsius, Nestor der deutschen Soziologie, Peter Roeder (1927-2011), schon Prof. in Hamburg, seit 1973 am MPI, aber in Marburg bei Froese und Elisabeth Blochmann und in engem Kontakt mit Klafki und en Geisteswissenschaften so- zialisiert, und der Psychoanalytiker Franz Wellendorf, damals noch als Soziologe an dem von Furck geleiteten Pädagogischen Zentrum tätig. Der gesamte einleitende Text, dem man auch den Übersichtsbeitrag von Wellendorf über „Ansätze zur erziehungswissenschaftlichen Theoriebildung in der $\mathrm{BRD}^{\prime 47}$ zurechnen darf, ist eine scharfe Auseinandersetzung mit der Tradition und im Ergebnis eine explizite Kritik am geisteswissenschaftlichen Programm, immer im Lichte der existenten Soziologien, eingeschlossen die kritische Theorie, die als „kritischdialektische Soziologie" eingeführt wird (S. 36f.). Die Soziologie als Referenz wird dann von Lepsius umfassend kontextualisiert und fortgesetzt in einer luziden soziologischen Kritik von „Bildung und Bildungsbegriff", die Roeder in einer Weise liefert, dass an diesem Grundbegriff der Tradition nicht viel theoretisch Bewahrenswertes bleibt (und man muss erinnern, dass Roeder schon Anfang der 1960er Jahre die spezifische Methodik der Tradition, die historischsystematische Analyse, am Beispiel Klafkis und seiner Rekonstruktion der Bildungstheorie destruiert hatte (Roeder, 1961, 1962; Klafki, 1961).

Die neun folgenden Bände zeigen, was das eigene Programm im Einzelnen bedeutet und zu bieten hat: Methodologisch, und das macht der So-

Comenius-Instituts. In: Annebelle Pithan (Hrsg.): Religionspädagoginnen des 20. Jahrhunderts. Göttingen 1997, S.342-356, u.a. mit der starken These, dass sie 1954 Leiterin wurde, weil man keinen renommierten Mann fand, "dem man die Aufbauarbeit zumuten und ihn entsprechend bezahlen konnte" (S. 346). Sie wurde auch erst 1964 als Direktorin bezeichnet, vorher als „"wissenschaftliche Sekretärin' und Mitarbeiterin des Vorstands" (ebd.).

${ }^{47}$ Franz Wellendorf unterscheidet in seinem Beitrag zum Goldschmidt-Band u.a. empirische Sozialwissenschaft, Normwissenschaft und Geisteswissenschaft (68ff.). 
ziologe Werner Mangold, noch ganz fern der Beschränkung auf statistische Fragen und psychologische Meßprobleme, wie sie aktuell nicht selten mit empirischer Bildungsforschung verwechselt werden, sondern breit in den sozialwissenschaftlichen Methoden, z.B. auch mit Einschluss von Beobachtung oder Gruppendiskussion. Peter Fürstenau liefert eine "Soziologie der Kindheit" , die theorie- und stilbildend wirkt und, wie Furck stolz rückblickend bemerkt, sogar das Feuilleton der FAZ aufmischt. C. Wolfgang Müller eröffnet mit „Jugend. Soziologische Materialien" ein Thema der Forschung, das bis heute bedeutsam bleibt und methodisch immer innovativ war (auch wenn Müller selbst dann eher sozialpädagogisch arbeitete). Für Klaus Mollenhauers „Jugendhilfe" muss man das vergleichbar sagen, auch wenn vielleicht seine Dissertation disziplinär und erziehungspolitisch für die Sozialpädagogik noch folgenreicher war (Mollenhauer, 1959; Aßmann, 2015). ${ }^{48}$ Wolfgang Schulz und Helga Thomas schreiben über Schulorganisation und Unterricht und lassen ahnen, was die Studien im Umkreis um Saul Robinsohn am MPI dann vertiefen werden. Hans-Günther Rolffs Analyse von "Sozialisation und Auslese durch die Schule" bestimmt für mehr als eine Generation die soziolo- gische Betrachtung des Bildungswesens und seiner Effekte auf soziale Schichten und in der Tradierung von Ungleichheit; der Autor präformiert damit unmittelbar auch die politische Argumentation, ja die kapitalismuskritische Agitation und Propaganda der Gesamtschulbefürworter in der Erziehungswissenschaft und in SPD und GEW ${ }^{49}$ - und die Bildungssoziologie löst sich nur mühsam aus diesen Denkfiguren. Wolfgang Nitsch, im Sozialistischen deutschen Studentenbund (SDS) und dessen Betrachtung der Universität erfahren, argumentiert über die "Hochschule“ (bleibt aber gegenüber der dann bald dominierenden Hochschuldidaktik eher randständig, trotz kontinuierlicher Arbeit im Feld (Nitsch, 1973). Johannes Schwerdtfegers Analysen über "Arbeit, Beruf, Betrieb" ist wichtig, erreicht aber nicht die Prominenz und langdauernde Wirkung, die man z.B. der Schule von Herwig Blankertz (Tenorth, 2000), selbst geisteswissenschaftlich sozialisiert, oder Martin Baethges Arbeiten zur Berufspädagogik. Das ist eine Wirkung schließlich, die man - mit Band X - Willy Strzelewicz für die Erwachsenenbildung und Johannes Aufermann und H. Bohrmann für die Analyse von Massenkommunikation wiederum nicht absprechen kann.

Die Herausgeber wissen, dass wei-

\footnotetext{
${ }^{48}$ Klaus Mollenhauer: Die Ursprünge der Sozialpädagogik in der industriellen Gesellschaft. Eine Untersuchung zur Struktur sozialpädagogischen Denkens und Handelns. Weinheim/Berlin 1959; für die Genese und Wirkung jetzt auch Alex Aßmann: Klaus Mollenhauer. Vordenker der 68er Begründer der emanzipatorischen Pädagogik. Eine Biografie. Paderborn 2015, bes. S. 101ff., bzw. S. $109 \mathrm{ff}$.

${ }^{49}$ Man lese die frühen Arbeiten von Klaus-Jürgen Tillmann, Herbert Stubenrauch oder Rolff selbst, wo kritische Pädagogik, Kapitalismuskritik und Gesamtschulpropaganda eine seltsame Mischung eingehen.
} 
tere Themen der Erziehung und der Konstitution des Selbst im Lebenslauf fehlen, sie wissen auch, dass weitere ideologiekritische Analysen notwendig sind. Man wird dennoch schwerlich bestreiten können, dass diese Veröffentlichungen eine Zäsur auf dem Weg der alten Pädagogik $\mathrm{zu}$ einer modernen Gesellschaftswissenschaft markieren und dass sie zugleich zeigen, dass man diesen Weg auch ohne umfassende Psychologisierung gehen kann, die ja später eher erfolgreich sein wird als eine soziologische Fundierung. Langfristig bedeutsam war diese soziologische Orientierung aber dennoch, theoretisch wie in den personenbezogenen Vernetzungen, allerdings mehr für kritische Erziehungswissenschaft und Bildungspolitik, schon mit Klaus Mollenhauer. Diese Richtung, scheinbar ganz fern von allen Konfessionalitätsproblemen, soll deshalb abschließend diskutiert werden, knapp im sozialen Kontext, vor allem unter der Frage, ob damit die Bedeutung des konfessionellen Faktors erhalten bleibt oder geschwunden ist.

\section{„KRItische PÄdagogiK" - BILdUNGSTHEORIE ALS „POLITISCHE Religion“}

Das Thema kann ich hier nur noch knapp abhandeln, kann es aber auch thesenhaft zuspitzen, weil die historiographischen und theoriekritischen Debatten innerhalb der Pädagogik über „kritische Erziehungswissenschaft" längst geführt worden sind, von Innen wie von außen. Sie wurden allerdings in dieser Innensicht meist geführt, ohne die Frage der Religion aufzunehmen, meist doch konzentriert auf die Ablösung von der geisteswissenschaftlichen Pädagogik und von den wissenschaftstheoretischen Kontroversen aus, die im soziologischen Positivismusstreit und in der Auseinandersetzung mit Wolfgang Brezinka vorgetragen wurden. Dann hat man Kritik an diesem Programm vorgetragen und Renaissancen propagiert - das kann ich hier auf sich beruhen lassen (Sünker und Krüger, 1999; Tenorth, 2016) ${ }^{50}$ Mein Zugang besteht nur in der Frage, ob sich diese Theorietradition und ihren aktuellen Vertreter, wie sie vor allem in der Heydorn-Nachfolge und im Umkreis von Andreas Gruschka zu finden sind, tatsächlich vollständig von den alten theologisch-religiösen Traditionen abgelöst haben, die im Protestantismus und latent noch in der Geisteswisssenschaftlichen Pädagogik und in der Bildungstheorie wirksam waren. Meine These ist, dass diese konfessionellen Traditionen fortwirken, und indem man den Modus ihres Überlebens und ihre eher latente Geltung

\footnotetext{
${ }^{50}$ Als Einstieg in die Debatte mag ein Band reichen: Heinz Sünker und Heinz-Hermann Krüger (Hrsg.): Kritische Erziehungswissenschaft am Neubeginn?! Frankfurt a.M. 1999; mein eigene Position findet sich hier auch: Die zweite Chance. Oder: Über die Geltung von Kritikansprüchen "kritischer Erziehungswissenschaft". ebd, S. 135-161; systematisch zur Diskussion von Programmen „kritischer" Erziehungswissenschaft jetzt auch: Heinz-Elmar Tenorth: „Kritische“ Bildungsforschung - Überlegungen zur Konjunktur eines Pleonasmus. In: Kritische Bildungsforschung. Hrsg: Cathleen Grunert, Anne Schippling, Nicolle Pfaff. Festschrift H.-H.Krüger, 2016 (i.Dr.).
} 
zeigt, erkennt man zugleich, dass diese Traditionen in einer spezifischen Form auch in der Pädagogik der DDR und sogar darüber hinaus fortgewirkt haben.

Das verbindende Glied zwischen den zunächst so scheinbar differenten Pädagogiken besteht in der bildungstheoretischen Argumentation und deren politisch-pragmatischen Implikationen, wie sie in der kritischen Erziehungswissenschaft vertreten werden. Es sind Denkformen einer politischen Theologie und einer scharfen Erlösungspädagogik, die ich hier identifiziere und von denen aus ich das Fortleben religiöser Tradition zeigen kann. Sie sind politisch, weil sie nicht auf die Individuen bezogen sind, wie das in den Wirkungsannahmen jansenistisch geht, sondern weil sie auf Annahmen über die kollektive Bedeutung von Bildungsprozessen beruhen. Nur um Einwände vorwegzunehmen: Ich suche die anhaltende Wirkung also nicht primär wissenschaftstheoretisch, obwohl man in der Abwehr einer "(wert)neutralen“ Wissen- schaft solche fortdauernden Gemeinsamkeiten auch finden könnte, genauso wie im Begriff der "Verantwortung“ der Pädagogik als einer praktischen Wissenschaft ${ }^{51}$ (Gruschka, 2012). Auch das gibt es, es ist mir zu wenig, die theologische Basis reicht tiefer und sie vereint sich mit der politischen Implikation.

Heinz-Joachim Heydorn ist das verbindende Glied zwischen kritischer Theorie, politischer, hier: sozialistischer, Programmatik und religiöser Fundierung (Titz, 1999; Koneff$k e, 2004)^{52}$ Seine Schriften werden explizit von der These aus geschrieben, dass die Arbeit der Pädagogik nur als „Erlösung“ angemessen verstanden ist. ${ }^{53}$ Damit ist auch die Arbeit des Lehrers nur als aktuelle Form der Leistung des biblischen Moses angemessen gedeutet, der das Volk Israel aus dem Exil ins gelobte Land führte. Exodus ist also die HintergrundMetapher, in der sich Religion und sozialistische, ja alle utopische Politik der Neuzeit zur Einheit bringen, Pädagogik ist das Vehikel der Einlö-

\footnotetext{
51 Diesen Begriff sucht z.B. Andreas Gruschka, wenn er betont, dass „,auch die wissenschaftliche Pädagogik eine advokatorische Verantwortung übernommen hat", und zwar ,im Interesse der nachwachsenden Generation“, sogar als „Bringepflicht zur Besserung“, in: A.G.: „Empirische Bildungsforschung" am Ausgang ihrer Epoche? In: BMBF (Hrsg.). Bildungsforschung 2020 - Herausforderungen und Perspektiven. Dokumentation der Tagung des Bundesministeriums für Bildung und Forschung vom 29.-30. März 2012. Bonn/Berlin 2014, S. 375-385, S. 385,.

52 Meine Heydorn-Interpretation folgt der Arbeit von Ewald Titz (1999): Bilderverbot und Pädagogik. Zur Funktion des Bilderverbots in der Bildungstheorie Heydorns. Weinheim, Basel; zu einem m.E. gescheiterten, weil in der Konzentration auf Politik für die Probleme der Denkform völlig blind - Versuch einer Verteidigung Heydorns gegen diese Analyse Gernot Koneffke (2004): Der Grund für die mögliche Befreiung von Herrschaft liegt im Diesseits - gegen die Theologisierung der kritischen Bildungstheorie. In: Pädagogische Korrespondenz H. 33, 2004/05, S. 15-41.

53 Deshalb ist es verwunderlich, dass Bühler u.a. in der Diskussion von "Erlöserfiguren“ nicht nur Heydorn ignorieren, sondern den gesamten Strang dieser pädagogisch-bildungstheoretischen Tradition ausblenden und sich stattdessen mehrheitlich auf brave Reformpädagogen konzentrieren.

${ }^{54}$ Gottfried Küenzlen: Der Neue Mensch. Ein Kapitel der säkularen Religionsgeschichte (1982); die Buchversion: Der Neue Mensch. Eine Untersuchung zur säkularen Religionsgeschichte der
} 
sung. Die Konstruktion des „Neuen Menschen“54 ( Küenzlen, 1982, 1994) ist dabei das Ziel, der sich aus der Verblendung löst und zum wahren Bewusstsein seiner wirklichen, zweiten Natur geführt wird (Bernhard, 2014) ${ }^{55}$, in Heydorns Worten: „Mit dem Begriff der Bildung ... begreift sich der Mensch als sein eigener Urheber, versteht er, dass ihm die Ketten, die das Fleisch aufschneiden, von Menschen angelegt sind, dass es eine Aussicht gibt, sie zu zerreißen. Bildung ist eine neue, geistige Geburt, kein naturalistischer Akt; mit ihr muß sich der Mensch noch einmal über sein Bewußtsein einholen, sich selbst repetieren." (Heydorn, 1979, 9-10.)

In sozialistischen Bildungsprogrammen generell, nicht nur bei Heydorn, ist das die systematisch zugrundeliegende Überzeugung. Entsprechend finden sich auch in der Bildungsreflexion und Bildungspolitik der DDR diese Formeln. Hier ist der „Kommunist" das Idealbild des neuen Menschen, der zu erziehen ist, damit er der Welt das Heil bringt. Die pädagogische Praxis kennt entsprechend auch ihren eigenen Kult der eigenen Heiligen: Gedenkecken für Thälmann in Schulen sind als Altarbilder gebaut, das Blutopfer des Stifters beglaubigt sein Erlösungswerk und schart die Gemeinde anbetend um ihn (etc.). Nicht zufällig wird vom Gedanken der „politischen Religion“ (Maier, 2003) aus aktuell der Versuch unternommen, die Praxis der totalitären Staaten des 20. Jahrhunderts zu verstehen und die funktionale Äquivalenz linker wie rechter Utopien zu belegen (Harten, 1997; Harten, Neirich und Schwerendt, 2006, Tenorth, 2003). Die Abwehr des sozialistischen Lagers, im Westen wie im Osten, gegen diese Interpretation rührt primär daher, dass damit strukturelle Vergleiche der sozialisatorischen Alltagspraxis und des jeweiligen Heldenkultus zwischen sozialistischen und nationalsozialistischen Staaten so eindeutig naheliegen, dass sie gefährlich für das eigene Selbstbild werden. Dabei gibt es, wenn man diese Praxis in allen anderen Aspekten zu vergleichen sucht, natürlich keine Gleichsetzung oder eine Identitätsbehauptung, sondern den Befund von Differenzen, wie ich $n$ erst vergleiche sichtbar machen. Dass aber letztlich die Konstruktion von Erziehungsstaaten die politische Realität war (Benner, Schriewer und Tenorth, 1998; Horn u.a., 2006) ${ }^{56}$ und "Gewalt", d.h. im Kontext des Erziehungssystems zumindest die systematisch ansetzende pädagogische Überwältigung der heranwachsenden die

Moderne. 2. Aufl. München 1994. Dort finden sich neben Nietzsche u.a auch die Psychoanalyse, die Jugendbewegungen des 20. Jahrhunderts und die russische "Revolution".

55 Die jüngste Aneignung, erneut im Modus des Bekenntnisses: Armin Bernhard, unter Mitarbeit von Sandra Schillings: Bewusstseinsbildung. Einführung in die kritische Bildungstheorie und Befreiungspädagogik Heinz-Joachim Heydorns. Hohengehren 2014.

56 Für diese Untersuchungsperspektive und ihre starke historische Plausibilität in rechten wie linken Diktaturen des 20. Jahrhunderts vgl. Dietrich Benner,Jürgen Schriewer undHeinz-Elmar Tenorth (1998, Hrsg.): Erziehungsstaaten. Historisch-vergleichende Analysen ihrer Geschichte und nationaler Gestalten. Weinheim, Basel, sowie Klaus-Peter Horn u.a. (2006, Hrsg.): Pädagogik im Militarismus und im Nationalsozialismus. Japan und Deutschland im Vergleich. Bad Heilbrunn. 
Konsequenz einer solchen Praxis ist, das kann man auch erkennen (Tenorth, 2003).

Bildungstheorie in den weltlichen Varianten und pädagogische Reflexion wiederum sind in diesen Kontext sowohl legitimatorisch wie operativ eingebaut. Als säkulare Theologie adaptieren sie die Denkform religiöser Welten und übersetzen sie in einen verweltlichten Kontext, operativ legitimieren sie den Zugriff auf die Subjekte. Insofern findet sich hier, und jetzt nicht nur in Deutschland, sondern in allen sozialistischen Traditionen (deren Denkform selbst sich neben den alten Utopien aber vor allem deutschen Quellen verdankt) diese Konvergenz des religiösen und des politischen Arguments in der pädagogischen Reflexion. Sie entfaltet sich als säkularisierte Religion, wo Bekenntnis und Milieubindung immer noch theoretische Geltungsgründe, methodische Skepsis und die Erfahrung kontraintentionaler Effekte entwerten kann. Das ist also nicht mehr nur ein deutsches Syndrom, sondern sozialistisches Erbe, gesamteuropäisch und weltweit anzutreffen, aber offenbar auch nach der Erfahrung des NS, des Gulag, von Pol Pot und der Regime nach 1945 und ihrer Unterdrückung oppositioneller und Freiheitsbewegungen in bestimmten Milieus bleibend attraktiv. Dieses Syndrom ist anscheinend immer und systematisch mit Erziehungsreflexion verbunden und mit der Liebe zur „Er- ziehungsdiktatur", die dieser Reflexion offenbar notorisch innewohnt.

In einer Bildungstheorie, die sich als Utopie, als politische Ideologie und als Religion des Pädagogischen versteht und als Erlösungstheorie begründet, scheinbar legitimiert durch das Versprechen einer neuen Welt und der Konstruktion eines neuen Menschen - in diesen Denkformen kommen die religiöse und die politische Tradition in der Moderne zur Einheit. Und natürlich: Distanz und Skepsis, kritische Prüfung und die Unterscheidung von Geltungsgründen in Theorie und Praxis haben hier so wenig Platz wie die dazu konträre, andere sozialphilosophische Grundüberzeugung der Moderne. Schon die liberale Grundprämisse z.B., dass Menschenrechte die Rechte des je individuellen Menschen sind, nicht die von Kollektiven oder von sozialen Bewegungen als Basis der Veränderung, wird als liberalistisch abgewertet (und die Kommunitarismus-Debatte wird nicht ernsthaft geführt, damit auch nicht die Kritik der Implikationen, die mit dem pädagogisch so beliebten Begriff der Gemeinschaft verbunden sind (vgl. Brumlik und Brunkhorst, 1993 $)^{57}$. Pädagogik kennt diese unterschiedlichen Referenzen aus ihrer eigenen Geschichte und sie muss sich entscheiden, theoretisch wie praktisch. Zumindest das kann man im Rückblick auf das 20. Jahrhundert lernen.

\footnotetext{
${ }^{57}$ Für eine erste, stark liberalismuskritische Präsentation und Diskussion der Probleme vgl. u.a. Micha Brumlik und Hauke Brunkhorst ( 1993, Hrsg.): Gemeinschaft und Gerechtigkeit. Frankfurt a.M. .
} 


\section{LITERATUR}

Aßmann, A. (2015): Klaus Mollenhauer. Vordenker der 68er-Begründer der emanzipatorischen Pädagogik. Eine Biografie. Paderborn.

Benner, Schriewer J. und Tenorth, H.-E. (1998, Hrsg.): Erziehungsstaaten. Historisch-vergleichende Analysen ihrer Geschichte und nationaler Gestalten. Weinheim, Basel.

Berg, C.,Herrlitz, H.-G. und Horn, K.-P. (2004): Kleine Geschichte der Deutschen Gesellschaft für Erziehungswissenschaft. Eine Fachgesellschaft zwischen Wissenschaft und Politik. Wiesbaden.

Bernfeld, S. (1925): Sisyphos oder die Grenzender Erziehung. Wien.

Bernhard, A. (2014): Bewusstseinrbildung. Einführung in die kritische Bildungstheorie und Befreiungspädagogik Heinz-Joachim Heyerdorns. Schneider Verlag, Hohengehren.

Brezinka, W. (1966): Die Krise der wissenschaftlichen Pädagogik im Spiegel neuer Lehrbücher. Zeitschrift für Pädagogik, 12. 53-88

Brezinka, W. (1967): Über den Wissenschaftsbegriff der Erziehungswissenschaft und die Einwände der weltanschaulichen Pädagogik. Zeitschrift für Pädagogik, 13. 135-168.

Brezinka, W. (2003): Pädagogik in Österreich. Die Geschichte des Faches an den Universitäten vom 18. bis zum Ende des 20. Jahrhunderts. In: Päd3agogik an den Universitäten Prag, Graz und Innsbruck. Wien.

Breyvogel, W. (1977): „Zum Ansatz des historischen Materialismus in der Lehrerforschung“. In: Heinemann, M. (Hrsg. 1977): Der Lehrer und seine Organisation. Stuttgart, 477-493.

Bühler, P., Bühler T. und Osterwalder, F. (2013, Hrsg.): Zur Inszenierungsgeschichte pädagogischer Erlöserfiguren. Haupt Verlag, Bern.

Büttemeyer, W. (1976, Hrsg.): Der Positivismusstreit in der deutschen Erziehungswissenschaft. München.

Brumlik, M., und Brunkhorst H.(1993, Hrsg.): Gemeinschaft und Gerechtigkeit. Frankfurt a.M.

Cloer, E. (1977): Sozialgeschichte, Schulpolitik und Lehrerfortbildung der katholischen Lehrerverbände im Kaiserreich und in der Weimarer Republik. Ratingen.

Diesterweg, F. A. W (1852): Kirchenlehre oder Pädagogik? (1852) In: ders.: Sämtliche Werke, Bd. IX, 375-472.

Dolch, J. (1933): Erziehung und Erziehungswissenschaft in Deutschland und Deutschösterreich von 1900 bis 1933. München.

Dolch, J. (1940): Lesebuch zur Erziehungswissenschaft.

Dolch, J. (1952): Aufgaben und Aufbau der Pädagogik, einer umstrittenen Wissenschaft. In: Röhrs, H. (1967, Hrsg.): Erziehungswissenschaft und Erziehungswirklichkeit. Frankfurt am Main, 123-136.

Eggersdorfer, F. X., Ettlinger, M. und Schröteler, J. (1928-1934): Handbuch der Erziehungswissenschaft. Kösel, München.

Elsenbast, V., Pithan A., Schreiner, P. und Schweitzer, F. (2004, Hrsg.): Wissen klären Bildung stärken. 50 Jahre Comenius Institut. Münster.

Eykmann, W. und Böhm, W. (2006, Hrsg.): Die Person als Maß von Politik und Pädagogik. Würzburg. 
Fischer, A. (1928): Die pädagogische Wissenschaft in Deutschland. In: Die neuzeitliche deutsche Volksschule. Bericht über den Kongreß Berlin 1928. Herausgegeben von der Kongreßleitung. Comenius Verlag, Berlin, 76-93.

Flitner, W. (1956): Das Selbstverständnis der Erziehungswissenschaft in der Gegenwart. Heidelberg (4. Aufl.1966).

Frost, U. (2006, Hrsg.): Unternehmen Bildung. Die Frankfurter Einsprüche und kontroverse Positionen zur aktuellen Bildungsreform. Paderborn 91-104.

Groothoff, H.-H. und Stallmann, M. (1970, Hrsg.): Neues Pädagogisches Lexikon. Kreuz Verlag, Stuttgart,Berlin.

Göttler, J. (1915/1947): System der Pädagogik. Verlag Kösel, München.

Gruschka, A. (2012): „Empirische Bildungsforschung" am Ausgang ihrer Epoche? In: BMBF (Hrsg.): Bildungsforschung 2020 - Herausforderungen und Perspektiven. Dokumentation der Tagung des Bundesministeriums für Bildung und Forschung vom 29.30. März 2012. Bonn,Berlin 2014, 375385.

Günther, K.-H. (1988): Über pädagogische Traditionen. Berlin

Haenisch, K. (1921): Neue Bahnen der Kulturpolitik. Aus der Reformpraxis der deutschen Republik. Stuttgart/Berlin.

Harten H.-C. (1997): Kreativität, Utopie und Erziehung. Grundlagen einer erziehungswissenschaftlichen Theorie sozialen Wandels. Opladen.

Harten, H.-C., Neirich, U. und Schwerendt, M. (2006): Rassenhygiene als Erziehungsideologie des Dritten
Reichs: Bio-bibliographisches Handbuch. Edition Bildung und Wissenschaft, Berlin.

Heinemann, M. (1977, Hrsg.): Der Lehrer und seine Organisation. Stuttgart.

Henz, H. (1964): Lehrbuch der systematischen Pädagogik. Freiburg.

Heydorn, H.-J. (1970): Über den Widerspruch von Bildung und Herrschaft. Frankfurt a. M.

Himmelstein, K. (2013): Das Konzept Deutschheit, Studien über Eduard Spranger. Peter Lang. Frankfurt am Main.

Hoffmann, V. (1977): „Der „Raussschmeißer Paragraph" - zur Geschichte einer Unvereinbarkeitsklausel im Deutschen Lehrerverein (LV) der Weimarer Republik“. In: Heinemann, M. (1977, Hrsg.): Der Lehrer und seine Organisation. Stuttgart.

Horn, K.-P. u.a. (2006, Hrsg.): Pädagogik im Militarismus und im Nationalsozialismus. Japan und Deutschland im Vergleich. Bad Heilbrunn.

Kanitz, O. F. (1925): Das proletarische Kind in der bürgerlichen Gesellschaft. Urania-Verlagsgesellschaft, Jena.

Kauder, P. (1925): Die PetzeltSchule in der deutschsprachigen Pädagogik: Personen — Kriterien - Programm - Entwicklung - Wirkungen. Spurensuche aus Sicht der erziehungswissenschaftlichen Wissenschaftsforschung. Vierteljahrsschrift für wissenschaftliche Pädagogik, 91. 217-242.

Kaufmann, H. B. u.a. (1991, Hrsg.): Kontinuität und Traditionsbrüche in der Pädagogik. Ein Gespräch zwischen den Generationen. Weinheim, Basel

Klafki, W. (1961): Zu Peter Roeders 
Bemerkungen. Die Deutsche Schule, 53. 582-593.

Koneffke, G. (2004): Der Grund für die mögliche Befreiung von Herrschaft liegt im Diesseits - gegen die Theologisierung der kritischen Bildungstheorie. Pädagogische Korrespondenz 33. 05. 15-41.

Kramp, W. (1970): Theorie der Schule. In: Speck, J. und Wehle G. (Hrsg.): Handbuch pädagogischer Grundbegriffe. München, Bd. 2. 529-589.

Kunst, H. und Grundmann, S. (1966, Hrsg.): Evangelisches Staatslexikon. Kreuz-Verlag, Stuttgart, Berlin.

Küenzlen, G. (1994): Der Neue Mensch. Eine Untersuchung zur säkularen Religionsgeschichte der Moderne. München.

Lamberti, M. (2002): The Politics of Education. Teachers and School Reform in Weimar Germany. Berghahn, New York.

Löwenstein, K. (1976, Hrsg. von Brandecker, F. und Feidel-Merz, H.): Sozialismus und Erziehung. Eine Auswahl aus den Schriften 1919-1933. Berlin/Bonn.

Maier, H. (2003, Hrsg.): Totalitarismus und Politische Religionen. Konzepte des Diktaturvergleichs. Band III: Deutungsgeschichte und Theorie. Paderborn.

Mertens, G., Frost, U., Böhm, W., Koch, L. und Ladenthin, V. (2007, Hrsg.): Handbuch der Erziehungswissenschaft. Görres-Gesellschaft, Paderborn.

Mollenhauer, K.(1959): Die Ursprünge der Sozialpädagogik in der industriellen Gesellschaft. Eine Untersuchung zur Struktur sozialpädagogischen Den- kens und Handelns. Weinheim, Berlin.

Morsey, R (1986): GörresGesellschaft. In: Görres-Gesellschaft (Hrsg.): Staatslexikon - Recht Wirtschaft Gesellschaft, Freiburg im Breisgau.

Müller, M. (2014): Das Deutsche Institut für wissenschaftliche Pädagogik 1922-1980. Von der katholischen Pädagogik zur Pädagogik von Katholiken. (Veröffentlichungen der Kommission für Zeitgeschichte. Paderborn.

Müller-Rolli, S. (1999, Hrsg.): Evangelische Schulpolitik in Deutschland 1918-1958. Dokumente und Darstellung. Göttingen 1999.

Nitsch, W. (1973): Die soziale Dynamik akademischer Institutionen. Weinheim, Basel.

Nohl, H. und Pallat, L. (1928-1933, Hrsg.): Handbuch der Pädagogik. Beltz, Langensalza.

Oelkers, J., Schulz, W. K. und Tenorth, H.-E. (1989,Hrsg.):Neukantianismus. Kulturtheorie, Pädagogik und Philosophie. Weinheim, Basel.

Osterwalder, F., Oelkers, J. und Tenorth, H.-E. (Hrsg.) (2003): Das verdrängte Erbe. Pädagogik im Kontext von Religion und Theologie. Weinheim, Basel.

Oesterwalder, F. (1999): Pädagogik - Ein Bekenntnis. Zur Architektur padagogische Diskurse und ihrer Geschichte. Neue Pestalozzi-Blätter. Zeitschrift für Historiographie, 5. 1. 21-25.

Pechar, H.(2012): Humboldt in der Massenuniversität? Vom Elend der neuhumanistischen Bildungsreligion. In: Kossek, B. und Zwiauer, C. (Hrsg.): Universität in Zeiten von Bologna. Wien, 69-82.

Pithan, A. (1997, Hrsg.): Religions- 
pädagoginnen des 20. Jahrhunderts. Göttingen, 342-356.

Rang, B. (1982): Pädagogische Geschichtsschreibung in der DDR. Frankfurt a.M., New York.

Roeder, P. M. (1961): Bemerkungen zu Wolfgang Klafkis. Untersuchungen über ,Das pädagogische Problem des Elementaren und die kategoriale Bildung. Die Deutsche Schule, 53. 572-581.

Roeder, P. M.: (1962): Zur Problematik der historisch-systematischen Methode. Die Deutsche Schule, 54. 3944.

Rühle, O. (1925/1969, Hrsg. von Werder, L. v. und Wolff, R.): Zur Psychologie des proletarischen Kindes. Frankfurt a.M.

Rühle, A. und Rühle, O. (1972): Erziehung und Gesellschaft. Berlin.

Sack, E. (1961): Die preußische Schule im Dienste gegen die Freiheit. Schulpolitische Kampfschriften. Ausgew. und eingel. von K.-H.Günther. Berlin.

Scheuerl, H. (1991): Zur Geschichte des Seminars für Erziehungswissenschaft. In: Krause, E., Huber, L. und Fischer, H. (Hrsg.): Hochschulalltag im "Dritten Reich". Die Hamburger Universität 1933-1945, Bd. 3, Teil II: Philosophische Fakultät rechtsund Staatswissenschaftliche Fakultät. Hamburg. 519-535.

Schwartz, H. (1928-1931, Hrsg.): Pädagogisches Lexikon in Verbindung mit der Gesellschaft für evangelische Pädagogik. Velhagen \& Klasing, Bielefeld.

Siemsen, A. (1926): Beruf und Erziehung. Berlin.

Spieler, K. J. (1930-1932, Hrsg.): Lexikon der Pädagogik der Gegenwart. Herder, Freiburg.
Stöhr, W. (1978): Lehrer und Arbeiterbewegung Entstehung und Politik der ersten Gewerkschaftsorganisation der Lehrer in Deutschland 1921 bis 1923. Marburg.

Sünker, H. und Krüger H.-H. (1999, Hrsg.): Kritische Erziehungswissenschaft am Neubeginn?! Frankfurt a.M.

Tenorth, H.-E. (1986): Transformationen der Pädagogik. 25 Jahre Erziehungswissenschaft in der Zeitschrift für Pädagogik.

Tenorth, H.-E. (1989): Die Last der Autonomie. Über Widersprüche zwischen Selbstbeschreibungen und Analysen des Bildungssystems seit dem 19. Jahrhundert. In: K.-E. Jeismann, K.E. (Hrsg.): Bildung, Staat, Gesellschaft im 19. Jahrhundert. Wiesbaden , 413431.

Tenorth, H.-E. (1990): Adorno, das Wetter und wir. Pädagogische Korrespondenz, 7. 41-49.

Tenorth, H.-E. (1996): Das Zentralinstitut für Erziehung und Unterricht. Außeruniversitäre Erziehungswissenschaft zwischen Politik, Pädagogik und Forschung. In: G. Geißler und U. Wiegmann (1996, Hrsg.): Außeruniversitäre Erziehungswissenschaft in Deutschland. Versuch einer historischen Bestandsaufnahme. Frankfurt a.M., 113-135.

Tenorth, H.-E. (1997): Reform Pädagogik - Religion. Der Evangelische Erzieher, 49. 376-384.

Tenorth, H.-E. (1999): Rettungslos der Religion verfallen? Fritz Osterwalder als Propagandist säkularer Pädagogik. Neue Pestalozzi-Blätter, 5. 2. 2123. 
Tenorth, H.-E. (1999): Schule - Religion - Zivilreligion. Zur weiteren Problematisierung eines jetzt schon schwierigen Verhältnisses. In: Ch.Th. Scheilke und F. Schweitzer (Hrsg.): Religion, Ethik, Schule. Bildungspolitische Perspektiven in der pluralen Gesellschaft. Münster, 175-186.

Tenorth H.-E. (2000): Karrierekatalysator und Theoriediffusion. Notizen zur Blankertz-Schule in der Erziehungswissenschaft. In: Adick, C., Kraul, M. und Wigger L. (Hrsg.): Was ist Erziehungswissenschaft? Festschrift für Peter Menck. Donauwörth , 97-126.

Tenorth, H.-E. (2003).: Erziehungsutopien zwischen Weimarer Republik und Drittem Reich. In: Hardtwig, W. (Hrsg.): Utopie und politische Herrschaft im Europa der Zwischenkriegszeit. München, 175-198.

Tenorth, H.-E. (2003): Pädagogik der Gewalt. Zur Logik der Erziehung im Nationalsozialismus. Jahrbuch für Historische Bildungsforschung, 9. 7-36.

Tenorth, H.-E. (2012): Bildung und Konfessionalität. Zur Geschichte einer deutschen Tradition oder: vom Bedeutungsverlust einer historisch bedeutsamen Struktur. In: Owetschkin, D. (Hrsg.): Tradierungsprozesse im Wandel der Moderne. Religion und Familie im Spannungsfeld von Konfessionalität und Pluralisierung. Klartext, Essen, 75-87.

Tenorth, H.-E. (2013): Erziehungswissenschaft - Kontext und Akteur reformpädagogischer Bewegungen. In: Keim, W. und Schwerdt, U. (Hrsg.).: Handbuch der Reformpädagogik in Deutschland. 2 Bde, Frankfurt am Main (usw. ) Teil 1: Gesellschaftliche Kontexte, Leitideen und Diskurse.
293-326.

Tenorth, H.-E. (2014): Müller, Markus: Das Deutsche Institut für wissenschaftliche Pädagogik 1922-1980. Von der katholischen Pädagogik zur Pädagogik von Katholiken. (Veröffentlichungen der Kommission für Zeitgeschichte. Theologische Literaturzeitung, 139. 12. 1476-1478.

Tenorth H.-E. (2016): „Kritische“ Bildungsforschung - Überlegungen zur Konjunktur eines Pleonasmus. In: Grunert, K., Schippling, A. und Pfaff, N. (Hrsg.): Kritische Bildungsforschung. (i.Dr.).

Tenorth, H.-E. und Brezinka, W. (2004): Wissenschaftliche Pädagogik im Spiegel ihrer ungelösten Probleme. Pädagogische Rundschau, 58. 453-465.

Tenorth, H.-E. und Oelkers, J. (2004): 50 Jahre „Zeitschrift für Pädagogik". Zeitschrift für Pädagogik, 50. 791-797.

Titz, E. (1999): Bilderverbot und Pädagogik. Zur Funktion des Bilderverbots in der Bildungstheorie Heydorns. Weinheim, Basel

Trube, M. (1977): „Zur Auseinandersetzung um Gewerkschaft und Streik im deutschen Lehrerverein (DLV) 1918-1922“. In: Manfred Heinemann (Hrsg.): Der Lehrer und seine Organisation. Stuttgart, 167-176.

Uhlig, C. (1979): Theoretische Auffassungen Paul Oestreichs zur Entschiedenen Schulreform. Jahrbuch für Erziehungs- und Schulgeschichte, 19.5367.

Uhlig, C. (2006): Reformpädagogik: Rezeption und Kritik in der Arbeiterbewegung. Frankfurt a.M. 